\title{
Chemotherapy enhances tumor cell susceptibility to CTL-mediated killing during cancer immunotherapy in mice
}

\author{
Rupal Ramakrishnan, Deepak Assudani, Srinivas Nagaraj, Terri Hunter, Hyun-Il Cho, \\ Scott Antonia, Soner Altiok, Esteban Celis, and Dmitry I. Gabrilovich \\ H. Lee Moffitt Cancer Center and Research Institute, Tampa, Florida.
}

\begin{abstract}
Cancer immunotherapy faces a serious challenge because of low clinical efficacy. Recently, a number of clinical studies have reported the serendipitous finding of high rates of objective clinical response when cancer vaccines are combined with chemotherapy in patients with different types of cancers. However, the mechanism of this phenomenon remains unclear. Here, we tested in mice several cancer vaccines and an adoptive $T$ cell transfer approach to cancer immunotherapy in combination with several widely used chemotherapeutic drugs. We found that chemotherapy made tumor cells more susceptible to the cytotoxic effect of CTLs through a dramatic perforin-independent increase in permeability to GrzB released by the CTLs. This effect was mediated via upregulation of mannose-6-phosphate receptors on the surface of tumor cells and was observed in mouse and human cells. When combined with chemotherapy, CTLs raised against specific antigens were able to induce apoptosis in neighboring tumor cells that did not express those antigens. These data suggest that small numbers of CTLs could mediate a potent antitumor effect when combined with chemotherapy. In addition, these results provide a strong rationale for combining these modalities for the treatment of patients with advanced cancers.
\end{abstract}

\section{Introduction}

Despite advances in the development of new chemotherapeutic drugs and improvements in radiation therapy, conventional cancer therapy often falls short of the goal of controlling tumor progression. Therapeutic cancer vaccines and adoptive $T$ cell transfer have long been considered very attractive therapeutic options in the treatment of cancer. However, despite identification of number of tumor-associated antigens that are recognized by CTLs, clinical trials of different cancer vaccines performed in recent years demonstrated a lack of clinical efficacy (1). It appears that cancer immunotherapy faces a number of challenges. They include the ability of vaccines to generate potent immune responses given the presence of numerous immunosuppressive factors, the ability of cytotoxic $\mathrm{T}$ cells to penetrate tumor parenchyma and recognize tumor-associated antigen, the correct choice of antigen for immunization, etc. It has become apparent that therapeutic cancer vaccines given as a single agent may not produce substantial clinical benefits, and combination with conventional methods of treatment will be necessary. However, the use of conventional cancer chemotherapy in combination with cancer vaccines was previously not considered as very attractive due to the potent immunosuppressive effect usually associated with chemotherapy. This paradigm was challenged in recent years by serendipitous observations made in a number of phase I/II clinical trials that reported high rates of objective clinical responses when cancer vaccines were combined with chemotherapy (2-6). These observations were made by several groups utilizing various cancer vaccines and different chemotherapeutic regimens in patients with diverse types of cancer (7). However, whether these findings represented a new paradigm or just anecdotal observations remained unclear. The mechanisms of the potential effect of com-

Conflict of interest: The authors have declared that no conflict of interest exists. Citation for this article: J Clin Invest. 2010;120(4):1111-1124. doi:10.1172/JCI40269. bined therapy remained unknown. In animal tumor models, it has been shown that conventional chemotherapy and radiation therapy can induce immune responses against antigens generated in dying tumor cells $(8,9)$. The paradox is that the types of chemotherapy that were used are known to suppress the immune system in cancer patients during standard treatment. Even in patients who benefited from combined treatment, chemotherapy inhibited antigenspecific $T$ cells generated by previously administered cancer vaccines (3). The effect of combined treatment in most patients was not long lasting, suggesting that effector cells may not remain functional for a long time. Therefore, understanding the cellular and molecular mechanisms of the effect of combined treatment is critically important for advancing the overall efficacy of this approach.

Here we report that several commonly used chemotherapeutic agents - paclitaxel (TAX), cisplatin (CIS), and doxorubicin (DOX) - sensitize tumor cells to CTLs by making tumor cells permeable to granzyme B (GrzB). This allowed antigen-specific CTLs to kill not only tumor cells expressing specific antigen but also the neighboring tumor cells that did not express those antigens. This effect was perforin independent and mediated via upregulation of mannose-6-phosphate (M6P) receptors on tumor cells. It explains how small numbers of CTLs could mediate a potent antitumor effect when combined with chemotherapy.

\section{Results}

Combination of immunotherapy and chemotherapy produces a potent antitumor effect. The main goal of cancer immunotherapy is to generate and deliver tumor-specific CTLs to tumor sites, with the prospect that CTLs will recognize and eliminate tumor cells expressing specific antigen (10). In this study, we used several different methods to generate tumor-specific CTLs to test the synergistic effect of immunotherapy and chemotherapy. Colon carcinoma MC38 is recognized by p53-specific CTLs due to overexpression of wild-type p53 
A

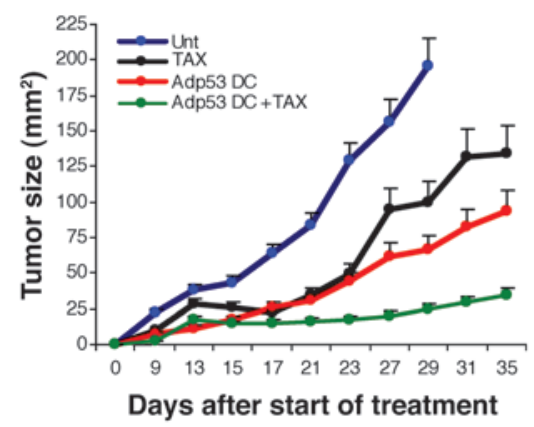

D
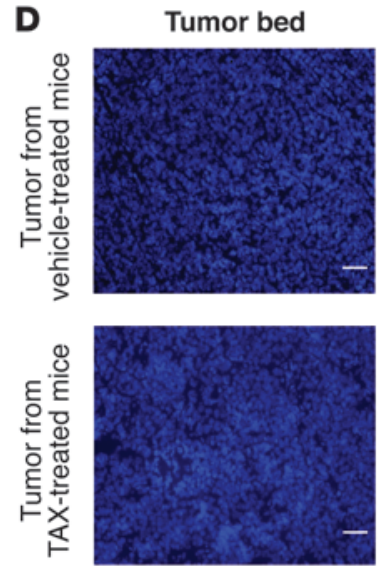

B

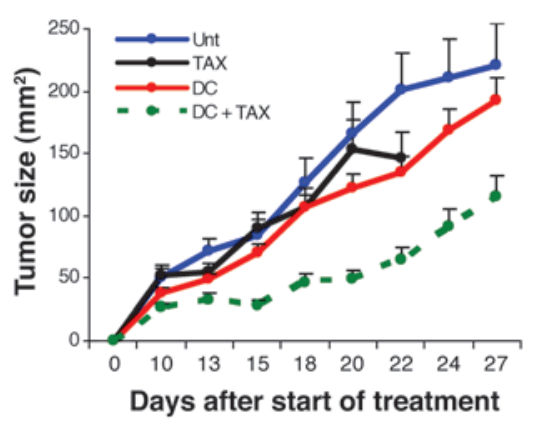

Days after start of treatment
C

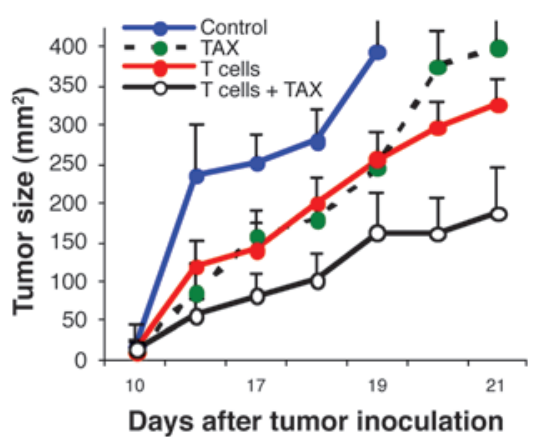

E

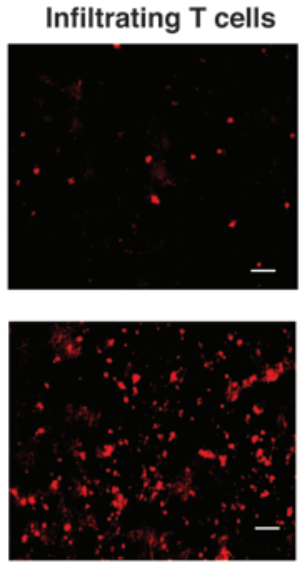

No. T cells infiltrating EG7 tumors in vehicle/TAX-treated mice

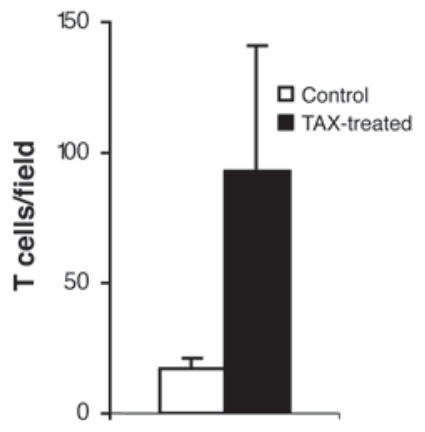

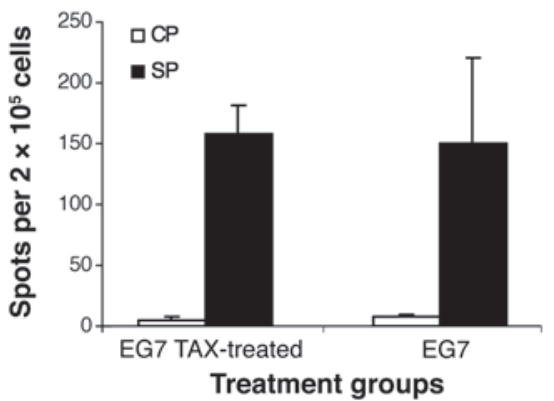

Figure 1

Combined effect of chemotherapy and immunotherapy. (A) Murine colon carcinoma tumors were established in C57BL/6 mice by s.c. injection of MC38 tumor cells. Treatment was started 5 days after tumor inoculation. Mice in treatment groups (DC, DC + TAX) were injected s.C. with $5 \times 10^{5} \mathrm{DCs}$ transduced with recombinant adenovirus containing the mouse wild-type p53 gene (Adp53). Immunizations were repeated twice at 7-day intervals. Treatment with TAX $(12.5 \mathrm{mg} / \mathrm{kg})$ was started 3 days after the second immunization. Tumor size was calculated by multiplying the 2 longest dimensions. $n=5$ mice group. The experiment was repeated twice with the same results. Unt, untreated. (B) Mammary carcinoma TUBO was established s.c. in BALB/c mice. The treatment times and intervals were the same as in A. DCs used for immunizations were loaded with $10 \mu \mathrm{g} / \mathrm{ml}$ Neu-derived peptide. $n=5$ mice group. The experiment was repeated twice with the same results. (C) T cells from mice immunized with OVA-derived peptide SIINFEKL were transferred to EG7 tumor-bearing C57BL/6 mice by i.v. injection of $5 \times 10^{6}$ cells. The treatment protocol for the treatment with TAX and adoptive transfer is described in Methods. $n=4$ mice group. The experiment was repeated once with the same results. (D) EG7 tumors were established s.c. in C57BL/6 mice. On day 16, the mice were treated with TAX (12.5 mg/kg) i.p. Three days later, they were administered $5 \times 10^{6}$ CMAC-labeled T cells from mice immunized with SIINFEKL. The tumors were removed 24 hours later, and cryosections were prepared. The slides were observed under a Leica fluorescence microscope, and 20 high-power $(\times 400)$ fields were counted per slide. Right: Number of T cells per tumor field in 3 mice per group. $P<0.05,2$-sided $t$ test. Scale bars: $5 \mu \mathrm{m}$. In A-D, data are shown as mean \pm SEM. (E) EG7 tumors were established by s.c. injection of $3 \times 10^{5}$ cells. When tumor reached $1.5 \mathrm{~cm}$ in diameter, $5 \times 10^{6}$ OT-T cells were injected i.v. in each mouse. After 3 days, half of the mice received $12.5 \mathrm{mg} / \mathrm{kg}$ BW TAX i.p. Splenocytes were collected 6 days later and restimulated with control (CP) or specific (SP) peptides. IFN- $\gamma$ production was evaluated by ELISPOT assay. The number of spots per $2 \times 10^{5} \mathrm{lymph}$ node cells are shown. Each point represents mean \pm SD of 6 replicates.

(11-13). Tumors were established s.c., and 3 days after tumor cell injection, mice were divided into different treatment groups. Vaccination of mice with DCs transduced with adenovirus containing full-length mouse wild-type p53 (Ad-p53) (14) resulted in a substantial delay in tumor progression (Figure 1A). In these initial experiments, we used TAX, a widely used chemotherapeutic agent. Treatment of mice with $12.5 \mathrm{mg} / \mathrm{kg}$ TAX alone delayed tumor growth, but tumor progression resumed soon after the treatment was discontinued. The combination of TAX and the DC vaccine potently suppressed tumor growth, which continued for at least 5 weeks after start of the treatment (Figure 1A). Thus, chemotherapy and immu- notherapy potentiated each other's effects in the model where each individual modality had antitumor activity. In the second model, TUBO mammary carcinoma tumors expressing the Neu oncogene (15) were established in BALB/c mice. Immunization was performed with DCs loaded with a Neu-derived peptide (p66) representing a CTL epitope (16). In this model, the selected dose of TAX had very little antitumor activity and vaccination alone only slightly delayed tumor growth (Figure 1B). However, the combination of the DC vaccine with TAX treatment resulted in a substantial antitumor effect in this model as well (Figure 1B). To test this approach in an experimental system with adoptive transfer of $\mathrm{T}$ cells, we used mice bear- 
A
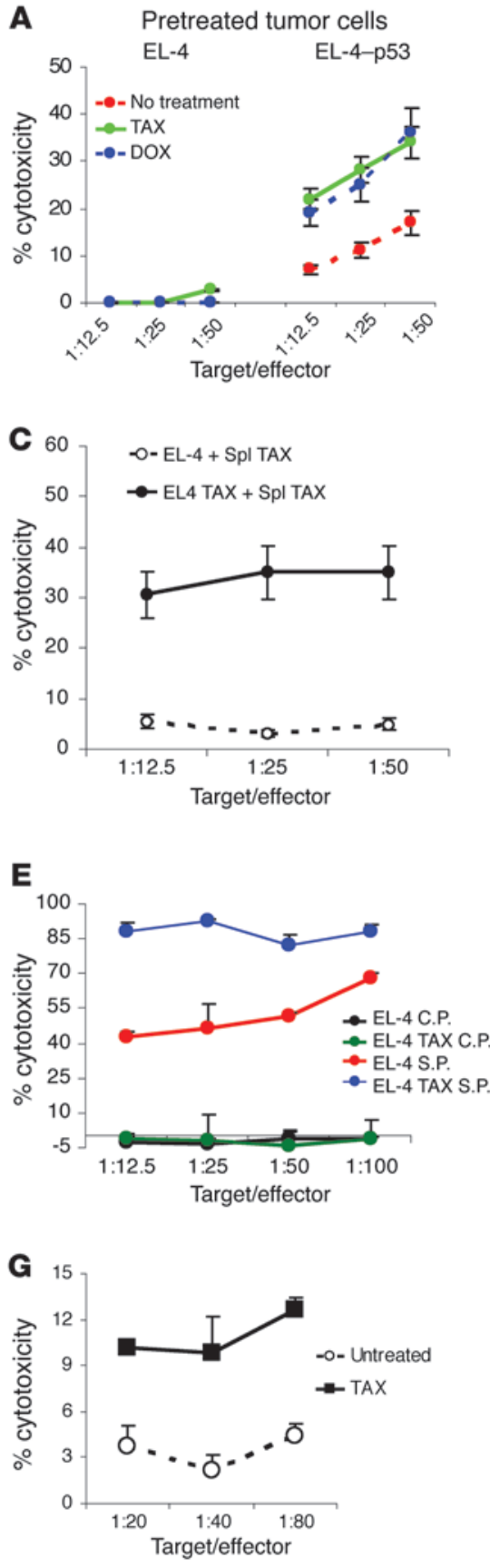

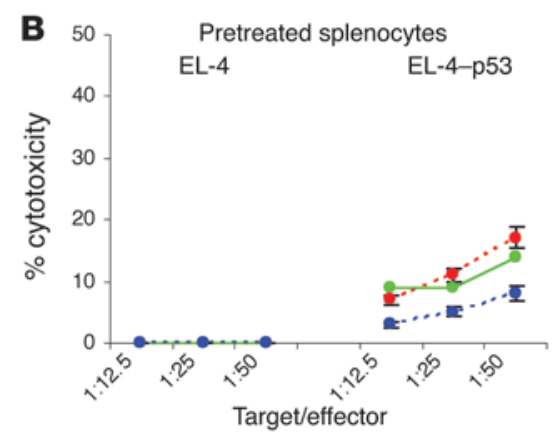

D
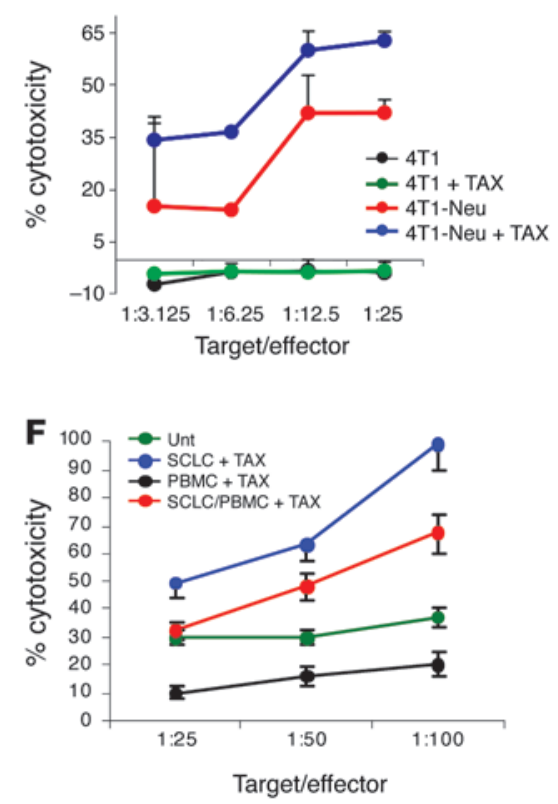

Figure 2

Chemotherapy sensitizes tumor cells to the cytotoxic effect of CTLs. (A) Tumor-free C57BL/6 mice were immunized with $\mathrm{K}^{\mathrm{b}}$-bound p53-derived peptide. Splenocytes were isolated, restimulated with the specific peptide for 6 days, and used as effectors in a CTL assay against EL-4 target cells loaded with specific (p53) or control peptides. EL-4 cells were pretreated overnight with $1.5 \mu \mathrm{g} / \mathrm{ml} \mathrm{DOX}$ or $12.5 \mathrm{nM}$ TAX. Standard 4-hour ${ }^{51} \mathrm{Cr}$-release assay was performed. For $\mathbf{A}-\mathbf{E}$, data are shown as mean \pm SEM. Each experiment was performed 3 times with the same results. (B) Splenocytes from immunized mice were pretreated overnight with DOX or TAX at doses described above and used as effectors against EL-4 target cells loaded with specific or control peptides in a $\mathrm{Cr}^{51}$ release assay. (C) Results of a CTL assay wherein both effectors and targets were pretreated with TAX as described above. Spl, splenocytes. (D) T cells from mice immunized with Neu-derived p66 peptide were used as effectors against either nonmodified 4T1 cells or 4T1-Neu cells transfected with Neu-expressing plasmid. The targets were pretreated with TAX for 18 hours. (E) T cells from $2 \mathrm{C}$-transgenic mice were used as effectors against EL-4 cells loaded with the specific (S.P.) or control (C.P.) peptides. The target cells were pretreated with TAX overnight. (F) Cytotoxicity against H332 SCLC cells was measured in duplicate in a standard 6-hour chromium release assay as detailed in Methods. PBMCs stimulated with SCLC tumor cells lysates were used as effectors. The viability of target cells and PBMCs in all tests was similar and greater than $85 \%$ at onset of assay. UNT, SCLC and PBMCs were not treated with TAX; SCLC+TAX, tumor cells were pretreated for 18 hours with $100 \mathrm{nM}$ TAX; PBMC+TAX, PBMCs were pretreated for 18 hours with $100 \mathrm{nM}$ TAX; SCLC/PBMC+TAX, tumor cells and PBMCs were treated with TAX. (G) Survivin-specific T cells were generated by 2 rounds of stimulation of mononuclear cells from $\mathrm{HLA}-\mathrm{A} 2^{+}$healthy volunteers as described elsewhere (18). The T cells were column purified and added to the tumor cells at indicated ratios. Primary tumor from $\mathrm{HLA}-\mathrm{A} 2^{+}$patient with non-SCLC cancer was used as target. The tumor cells were treated with 50 nM TAX 18 hours prior to ${ }^{51} \mathrm{Cr}$ release assay. ing EG-7 tumors (EL-4 cells expressing chicken OVA). T cells from mice immunized with OVA-derived peptide (SIINFEKL) were used for adoptive transfer (Supplemental Figure 1; supplemental material available online with this article; doi:10.1172/JCI40269DS1). $\mathrm{T}$ cells or TAX alone caused a decrease in tumor growth. However, this effect was substantially more pronounced when $\mathrm{T}$ cell transfer and TAX administration were combined (Figure 1C).

To evaluate the ability of $\mathrm{T}$ cells to penetrate into tumor parenchyma, we labeled OVA-specific T cells with the fluorescent tracker CMAC and transferred them into EG-7 tumorbearing mice 3 days after TAX injection. Tumors were excised
24 hours after $\mathrm{T}$ cell administration. Only a few tumor-infiltrating $\mathrm{T}$ cells were present in the mice not treated with TAX. In contrast, in mice treated with TAX, this number was significantly higher (Figure 1D).

We tested the possibility that TAX could improve antigen-specific response in tumor-bearing mice. OT-1 T cells were transferred into EG-7 tumor-bearing mice, followed 2 days later with injection of $12.5 \mathrm{mg} / \mathrm{kg}$ TAX. The antigen-specific response of splenocytes was evaluated 6 days after TAX injection in an IFN- $\gamma$ ELISPOT assay. No statistically significant $(P>0.1)$ differences between TAX-treated and control mice were found (Figure 1E). This was consistent with 

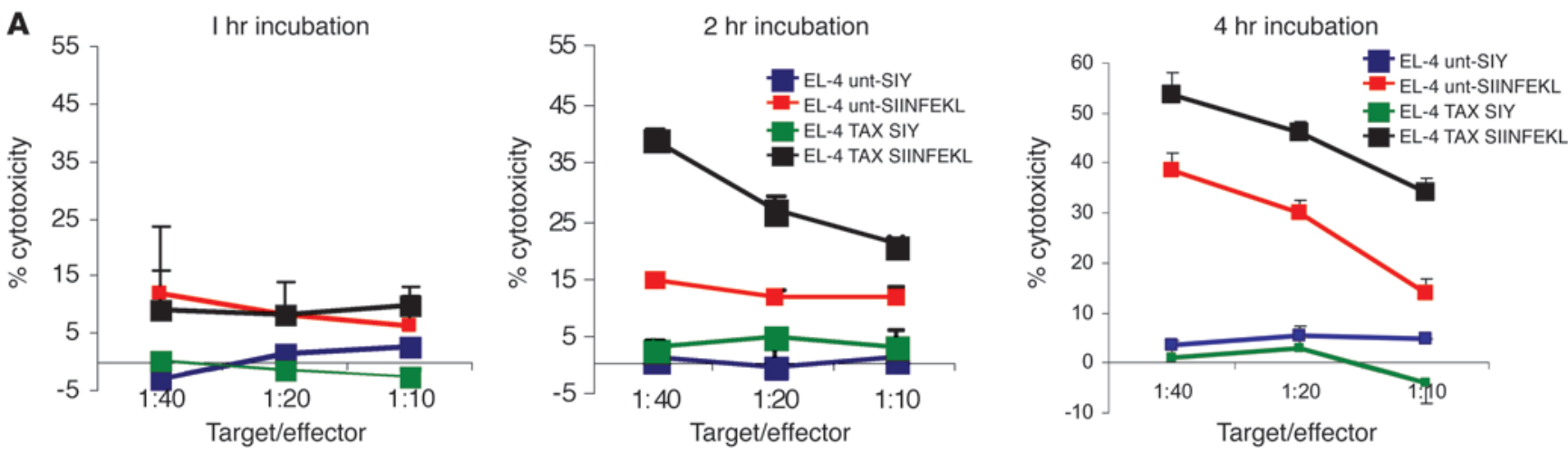

B
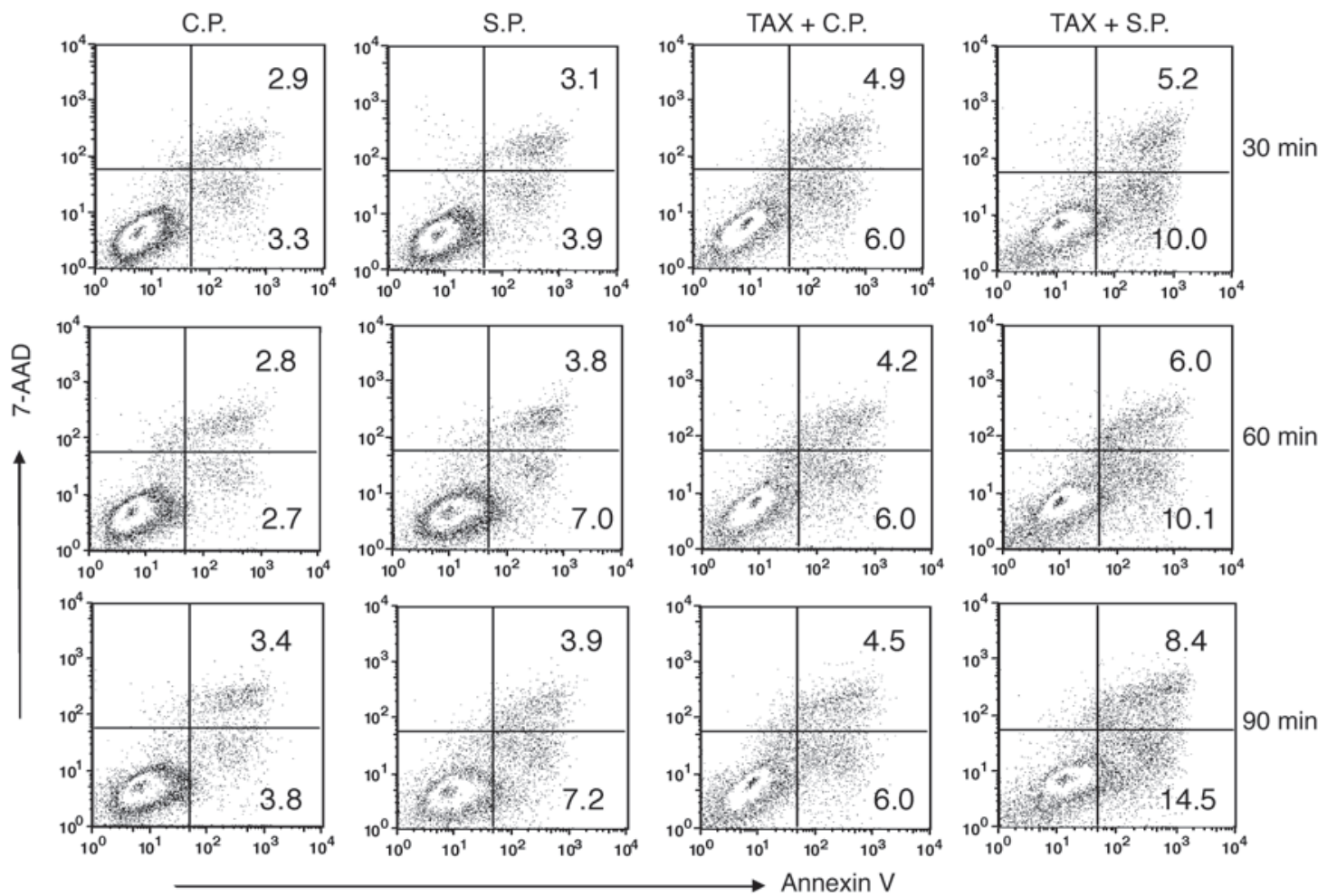

\section{Figure 3}

Kinetics of apoptosis in combination therapy. (A) OT-1 T cells were mixed with EL-4 cells that had been loaded either with specific SIINFEKL or a control SIYRYYG (SIY) peptide. Target cells were either pretreated with TAX overnight or were left untreated. Chromium release assay was performed in duplicate 1, 2, and 4 hours after start of the incubation. The $2 \mathrm{~h}$ and $4 \mathrm{~h}$ data were obtained from different reproducible experiments. (B) For the detection of early apoptosis, the effector OT-1 T cells were labeled with DDAO-SE and incubated with target EL-4 cells loaded either with specific or control peptides at a 20:1 ratio. After the indicated incubation time, cells were stained with Annexin V-FITC and 7-AAD. The proportion of Annexin Vpositive cells was measured within the population of tumor cells by flow cytometry. Typical result of 1 of 3 performed experiments is shown.

the previous observation that injection of TAX induced defects in antigen-nonspecific functions of $T$ cells (17) and suggested that the antitumor effect of this combined treatment was not likely due to positive effect of TAX on tumor-specific $\mathrm{T}$ cells.

Chemotherapy sensitizes tumor cells to the cytotoxic effect of CTLs. Next we investigated whether the chemotherapy agents can affect the susceptibility of tumor cells to the lytic effect of CTLs. Antigenspecific $T$ cells were generated by immunizing naive C57BL/6 mice with Ad-p53 DCs. Splenocytes were restimulated with p53derived $\mathrm{H}_{2} \mathrm{~K}^{\mathrm{b}}$ peptide (KYMCNSSCM) for 5 days and then used as effectors in CTL assays. As a target, we used EL-4 tumor cells loaded with control or specific peptide. We evaluated the effect of 3 chemotherapeutic drugs with different mechanism of action: TAX, DOX, and CIS. Tumor cells were pretreated with TAX or DOX for 18 hours. All chemotherapeutic agents have a delayed toxic effect on target cells. The kinetics of this effect depends on the dose of the drug. In preliminary experiments, we selected the doses of the drugs that did not induce more than $5 \%$ apoptosis of tumor cells after 18-24 hours of culture. Substantial cell death was observed only after 48 hours of treatment (data not shown). This was done to exclude a direct cytotoxic effect of the compounds. In all experiments, the level of spontaneous chromium release of tar- 

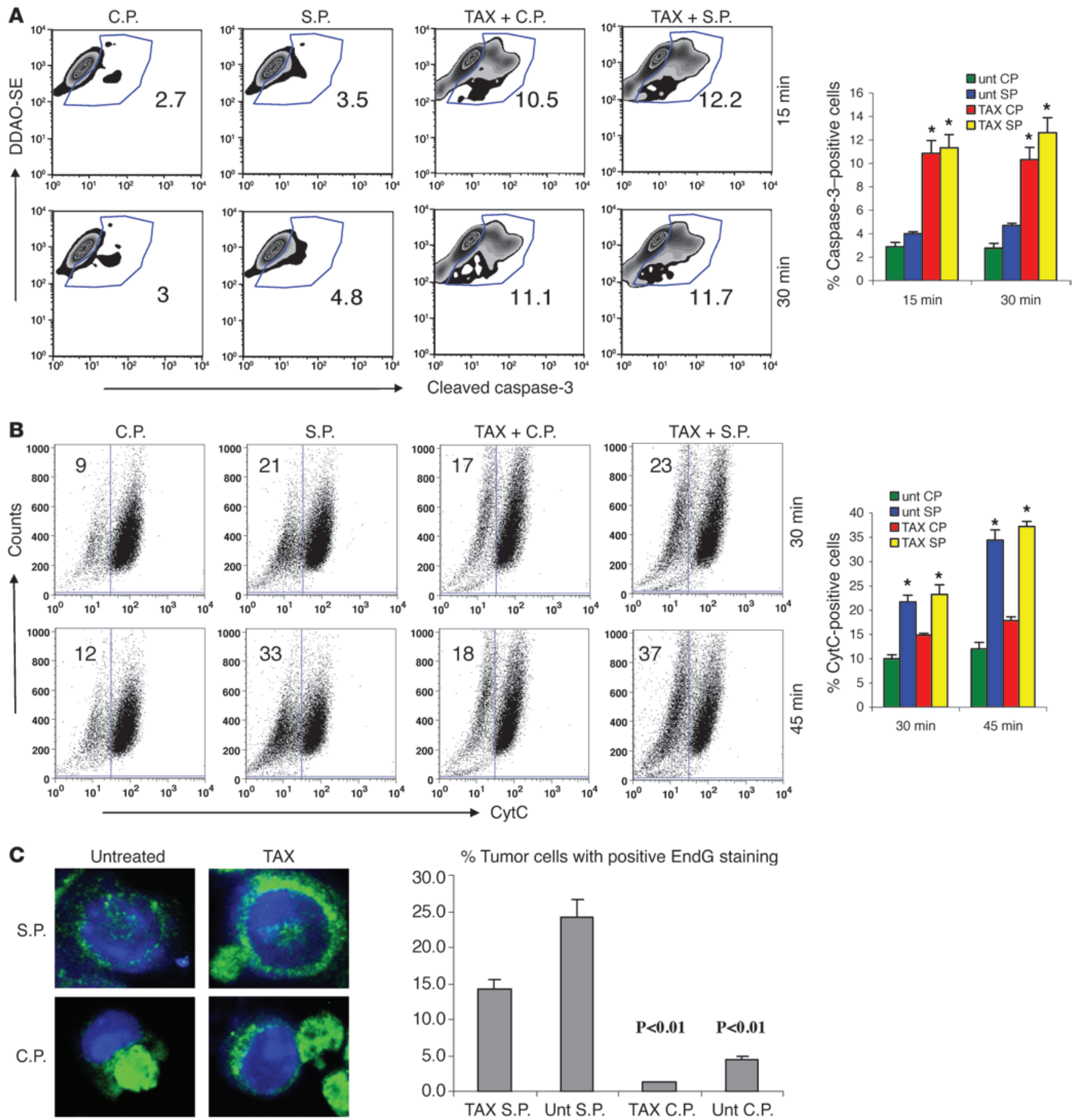

Figure 4

Mechanism of apoptosis induced by CTLs and TAX. (A) Cleaved caspase-3 in tumor cells. Untreated and TAX-treated EL-4 targets were labeled with DDAO-SE and loaded with control or specific peptide. The tumor cells were incubated with purified OT-1 T cells at a 1:10 ratio. After the indicated incubation time, cells were permeabilized and labeled with PE-conjugated antibody against cleaved caspase-3. Target cells were gated, and the levels of cleaved caspase- 3 in the target cells were analyzed by flow cytometry. ${ }^{*} P<0.05$ versus untreated EL- 4 cells. (B) CytC in tumor cells. Experiments were performed as described in Figure $2 \mathrm{~B}$. The cells were permeabilized, fixed, and labeled with anti-CytC antibody. ${ }^{*} P<0.05$ versus untreated EL-4 cells. In A and B, typical results of 1 experiment (left) and mean \pm SEM of 5 experiments (right) are shown. (C) EndG in tumor cells. EL-4 cells were treated with TAX for 18 hours and loaded with control or specific peptides as described above. OT-1 T cells were labeled with Po-Pro-3 iodide to distinguish them from tumor cells and incubated with target EL-4 cells at a 10:1 ratio for 1 hour. The cells were fixed and stained with anti-EndG antibody and DAPI. Visualization of the staining in the nuclei was performed using a Leica confocal microscope. The images were analyzed with Image Pro Plus 6.2 software. One hundred tumor cells were counted, and the proportion of those with positive nuclear staining was determined. The $P$ values were calculated using Fisher 2-tailed exact test. Data are presented as mean \pm SEM. 
get cells treated with chemotherapeutic agents was similar to that of untreated cells. Tumor cells were washed after drug treatment, loaded with control or specific peptides, and then cultured at different ratios with effector cells. CTLs alone displayed moderate but clear specific cytotoxicity. Pretreatment of tumor cells with TAX or DOX significantly increased CTL-mediated cytotoxicity of target cells loaded with the p53 peptide but had no effect on the levels of cytotoxicity obtained with targets loaded with an irrelevant control peptide (Figure 2A). To assess whether chemotherapeutic drugs could directly enhance the cytotoxic activity of CTLs, we pretreated the CTLs for 18 hours with the same doses of TAX and DOX and measured their cytolytic activity against peptide-pulsed EL-4 target cells. Pretreatment of CTLs with TAX did not affect the specific cytotoxicity, whereas DOX reduced it (Figure 2B). Importantly, the synergistic cytotoxic effect remained when both CTLs and tumor cells were pretreated with TAX (Figure 2C), which would reflect the in vivo conditions of treatment with chemotherapy. TAX also sensitized EL-4 tumor cells loaded with OVA-derived peptide (SIINFEKL) but not with control peptide to OT-1 CTLs (Figure 3A). Similar results were obtained in experimental models where OT-1 CTLs were used against specific peptide-loaded EL-4 target cells pretreated with DOX or CIS (Supplemental Figure 2, A and B). Importantly, pretreatment of OT-1 CTLs with TAX, DOX, or CIS substantially reduced their ability to kill target cells loaded with specific peptide (Supplemental Figure 2C). TAX sensitized tumor cells to CTLs also in the models where CTLs specific for Neu-derived peptide p66 were used against 4T1 tumor cells transfected with Neu-expressing plasmid (Figure 2D); and where 2C-transgenic T cells that recognize SIYRYYGL peptide were used against peptide-loaded EL-4 target cells (Figure 2E).

To test whether a similar phenomenon can be observed in humans, we used CTLs from allogeneic donors as effectors against the small cell lung cancer (SCLC) cell line H332. Pretreatment of tumor cells with TAX dramatically increased CTL cytotoxicity (Figure 2F). A slightly less-pronounced effect was observed when both PBMCs and SCLC were treated with TAX. In contrast, pretreatment of PBMCs with the same drug substantially reduced cytotoxicity (Figure 2F). In a different experimental system, we used primary tumor samples collected from patients with nonSCLC during surgical resection. Patients' tumors were propagated in nude mice, and 1 HLA-A2-positive tumor was used as target in CTL assay. Survivin-specific T cells were generated by repeated stimulation of mononuclear cells from HLA-A2-positive healthy donor with DCs transduced with mutant survivin as described previously (18). Overnight treatment of tumor with a selected dose of TAX $(50 \mathrm{nM})$ did not affect the level of spontaneous ${ }^{51} \mathrm{Cr}$ release (data not shown). However, it significantly increased cytotoxicity when tumor cells were cultured together with T cells (Figure 2G). These results replicated the effect observed in animal models. Overall, these data indicate that chemotherapy sensitizes tumor cells to the cytotoxic effect of CTLs.

Mechanism of synergy between chemotherapy and CTLs. To investigate the kinetics of interaction between CTLs and tumor cells in real time, OT-1 T cells were labeled with CFDA-SE cell tracer and mixed at 10:1 ratio with target EL-4 cells loaded with control or specific peptides in the presence of propidium iodide (PI). Cells were observed using live cell imaging. One hour incubation was not sufficient for CTLs to kill nontreated tumor cells loaded with specific peptide (data not shown). However, if the peptide-pulsed EL-4 cells were pretreated with TAX, the cytotoxic effect of CTLs was easily detectable (Supplemental Video 1 ). In ${ }^{51} \mathrm{Cr}$ cytotoxicity assay, 2 hours incubation of target cells with CTLs was sufficient to detect strong peptide-specific killing if target cells were pretreated with TAX (Figure 3A). This accelerated kinetic effect was further confirmed using an apoptosis assay with Annexin-V/7-AAD staining. In contrast to nontreated target cells, where little apoptosis was evident at early time points, EL-4 cells that were pretreated with TAX were susceptible to apoptosis induced by specific CTLs as early as 30 minutes of incubation. This effect became prominent after 90 minutes of incubation with the drug (Figure 3B). A similar effect was observed when EL-4 target cells were pretreated with DOX or CIS (Supplemental Figure 3).

Next we evaluated the mechanism of apoptosis caused by combination of chemotherapy and CTLs. Pretreatment of tumor cells with TAX caused a substantial increase in cleaved caspase- 3 in tumor cells. However, this effect was not enhanced by the addition of CTLs (Figure 4A). In contrast, CTLs substantially increased the proportion of cytochrome $c$-positive (CytC-positive) tumor cells. TAX, however did not enhance this effect (Figure 4B). CytC release ultimately results in activation of caspase- 3 . Therefore, we also evaluated release and nuclear localization of endonuclease $G$ (EndG), a mitochondrial proapoptotic protein that is involved in caspase-independent DNA degradation (19). CTLs caused a dramatic increase in the proportion of specific peptide-loaded tumor cells with nuclear localization of EndG as compared with cells loaded with the control peptide. TAX did not affect nuclear accumulation of EndG (Figure 4C). Thus, it appears that TAX and CTLs affect different components of the apoptotic pathway during the initial interaction between CTLs and tumor cells.

Chemotherapy makes tumor cells permeable for GrzB. CTLs mediate their cytotoxic effect via 2 major pathways: Fas/FasL and perforin/GrzB. Treatment of EL-4 or MC38 tumor cells as well as splenocytes with selected doses of TAX, CIS, or DOX did not significantly change the level of expression of Fas or FasL (Supplemental Figure 4), suggesting that it is unlikely that chemotherapy sensitizes tumor cells to CTLs via upregulation of these molecules. To assess the role of GrzB, we loaded EL-4 cells pretreated with TAX with control or specific peptides and labeled them with the dye CellTracker Blue CMAC. Tumor cells were incubated with OT-1 $\mathrm{CD}^{+}$CTLs at a 1:10 ratio, followed by intracellular staining with anti-GrzB antibody. Within 15 minutes of incubation, the proportion of GrzB-positive tumor cells was more than 3-fold higher when tumor cells were pretreated with TAX and loaded with specific peptide compared with untreated tumor cells (Figure 5A). These results suggested that pretreatment of tumor cells with TAX allowed for fast and more effective penetration of GrzB into tumor cells. To verify these observations, we used recombinant mouse GrzB, which was modified to block its protease activity and thus would not cause apoptosis of the cells. EL-4 tumor cells were pretreated with TAX, DOX, or CIS and then incubated with recombinant GrzB for 2 hours. Cells were then stained with antiGrzB antibody. On comparison with nontreated cells, treatment of tumor cells with all 3 drugs resulted in a substantial (more than 4-fold) increase in the intracellular GrzB level (Figure 5B). Similar experiments were performed with 3 different human SCLC lines and human recombinant GrzB. All 3 drugs caused more than a 5-fold increase in intracellular GrzB levels in these cell lines (Figure 5C). To verify a possible role of GrzB in the cytotoxic effect of CTLs combined with TAX, we treated cells with specific inhibitor of GrzB Z-AAD-CMK. Blocking of GrzB activity 
A
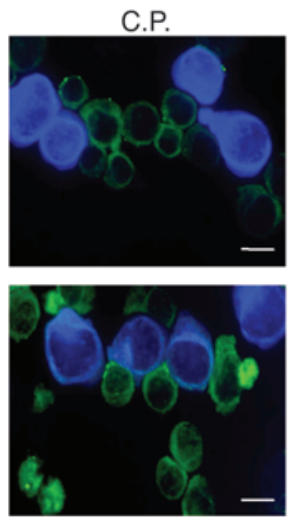

$\mathbf{B}$

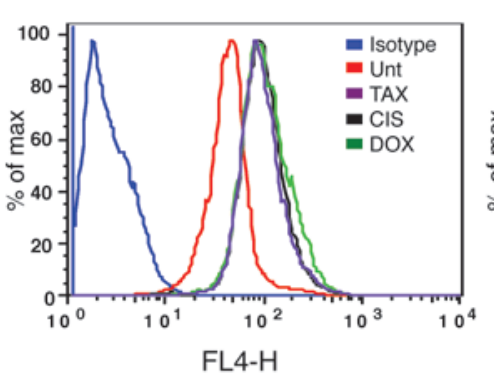

S.P.
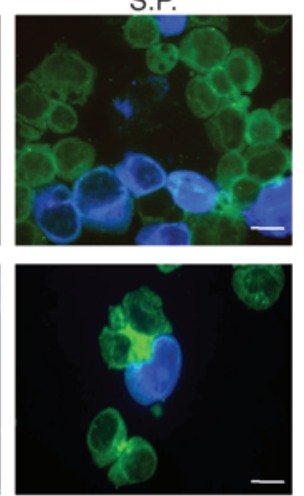

TAX + C.P.
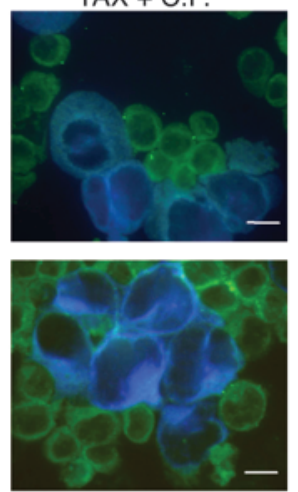

TAX + S.P.
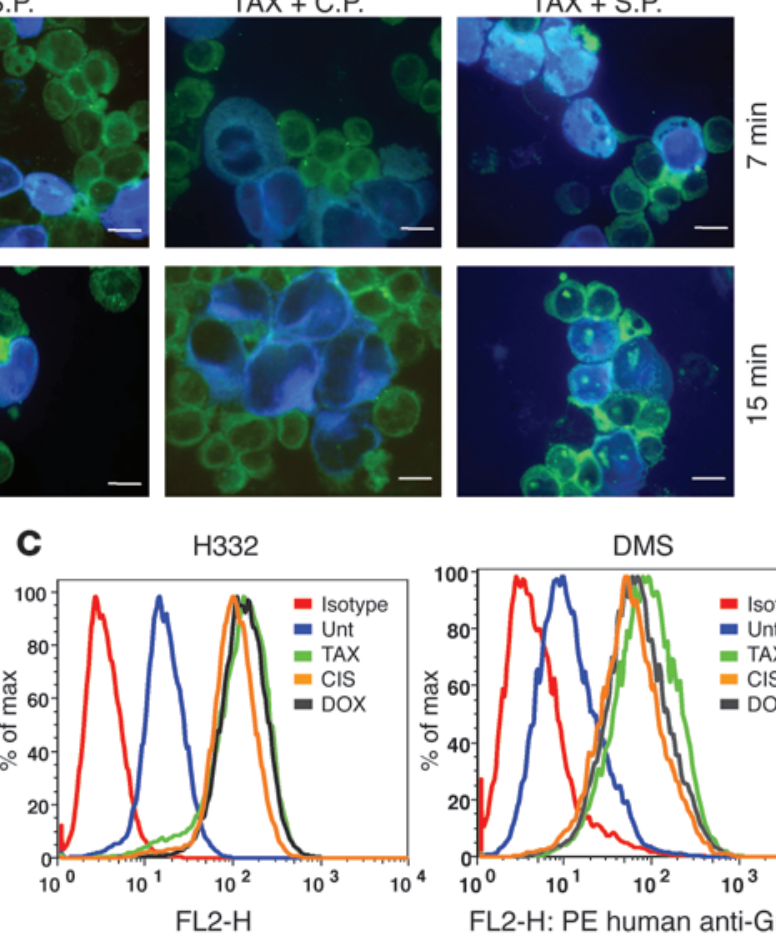

DMS

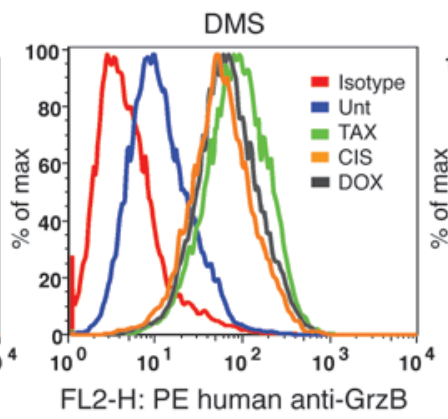

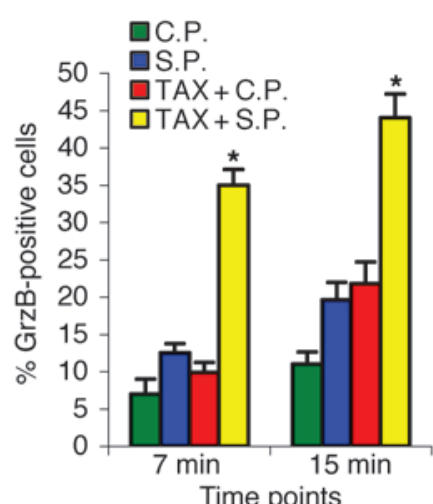

Time points
D
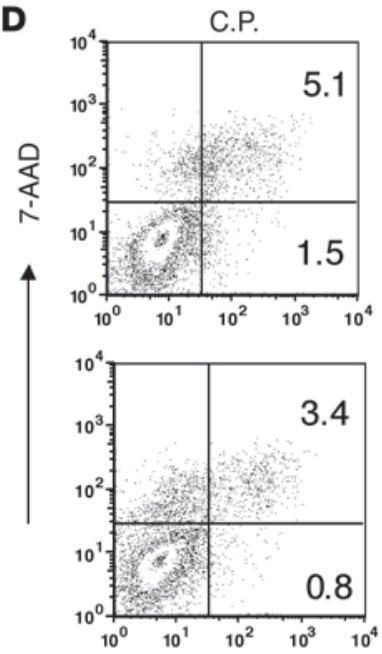

S.P.
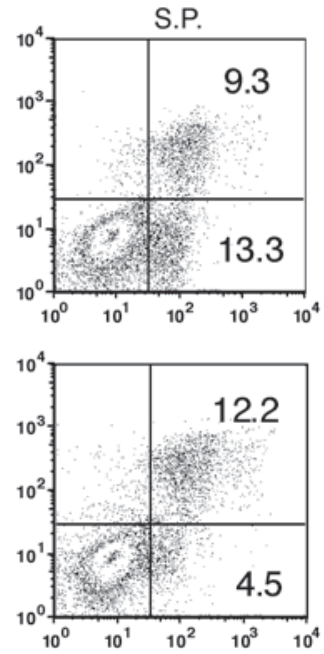
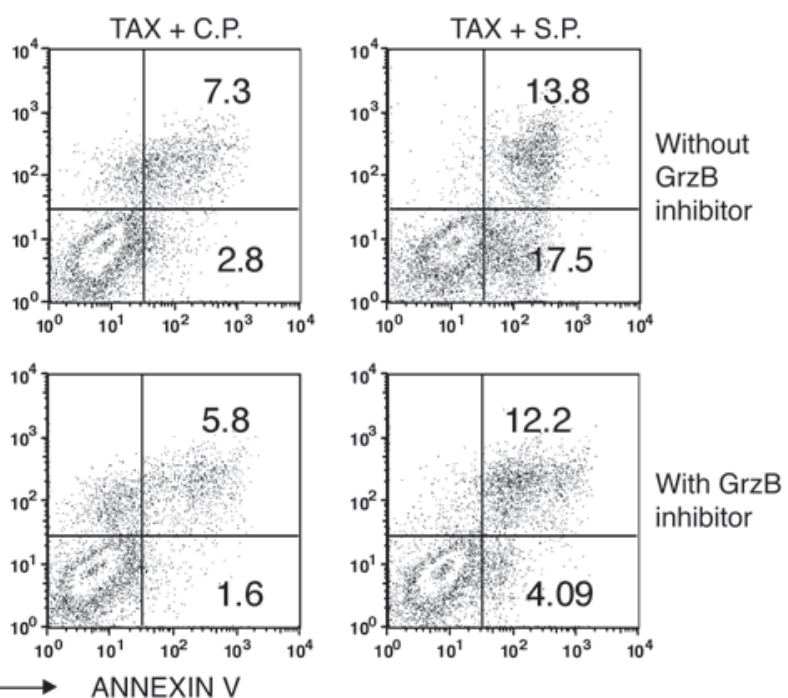

Figure 5

The effect of chemotherapy on permeability of tumor cells to GrzB. (A) EL-4 cells were treated with TAX and loaded with control or specific peptides as described above. Cells were labeled with CMAC and mixed with OT-1 cells at a 1:10 ratio and incubated for 7 and 15 minutes, then fixed and stained with anti-GrzB monoclonal antibody, followed by FITC-conjugated secondary antibody. The proportion of GrzB+ cells among blue target cells was calculated in triplicate by counting 200 target cells. Scale bars: $10 \mu \mathrm{m}$. Data represent mean \pm SEM of 4 experiments. ${ }^{*} P<0.05$ versus untreated tumor cells loaded with control peptide. (B) CMAC-labeled TAX-, DOX-, or CIS-treated EL-4 cells were incubated with $1 \mu \mathrm{g} / \mathrm{ml}$ recombinant mouse GrzB for 30 minutes. Cells were fixed and stained with anti-mouse GrzB antibody. The presence of GrzB was detected by flow cytometry. Histogram overlays represent isotype control, untreated EL-4 cells, and TAX-, CIS-, or DOX-treated cells. Each experiment was repeated 3 times with the same results. (C) The above experimental procedure was used for detecting the presence of GrzB+ cells in human cell lines. Human recombinant GrzB and PE-conjugated anti-human GrzB antibody were used. Tumor cells were treated overnight with $12.5 \mathrm{nM}$ TAX, $25 \mathrm{ng} / \mathrm{ml}$ DOX, or $25 \mathrm{ng} / \mathrm{ml}$ CIS. These doses did not decrease cell viability after overnight treatment by more than $5 \%$ but significantly reduced the cell growth of tumor cells. After 48 hours, these doses caused apoptosis in more than $90 \%$ of cells. Isotype control IgG was used in all samples and showed similar values. Isotype control of TAX-treated cells is shown. Histogram overlays represent isotype, untreated tumor cells, and TAX-, CIS-, or DOX-treated cells. Each experiment was repeated twice with the same results. (D) OT-1 T cells were pretreated with GrzB inhibitor I (Z-AAD-CMK) prior to incubation with EL-4 cells. The target cells were labeled with CMAC; the effectors were labeled with DDAO-SE. The target population was assessed for apoptosis using Annexin V-FITC/7AAD staining and analyzed using flow cytometry. Apoptosis was measured among tumor cells. Data represent 2 experiments with the same results. 
A EL-4 tumor cells

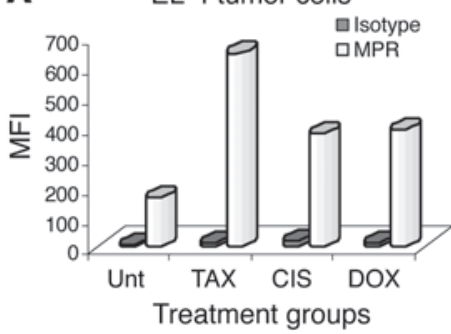

4T1 tumor cells

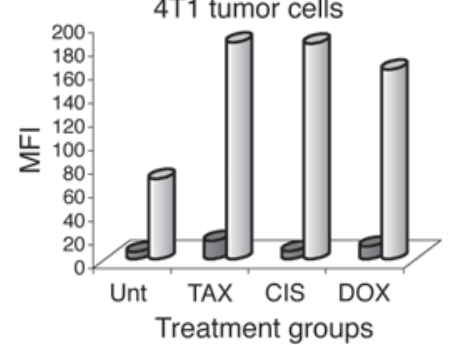

86M1 cells

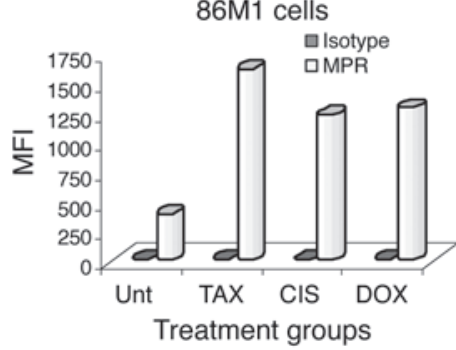

B
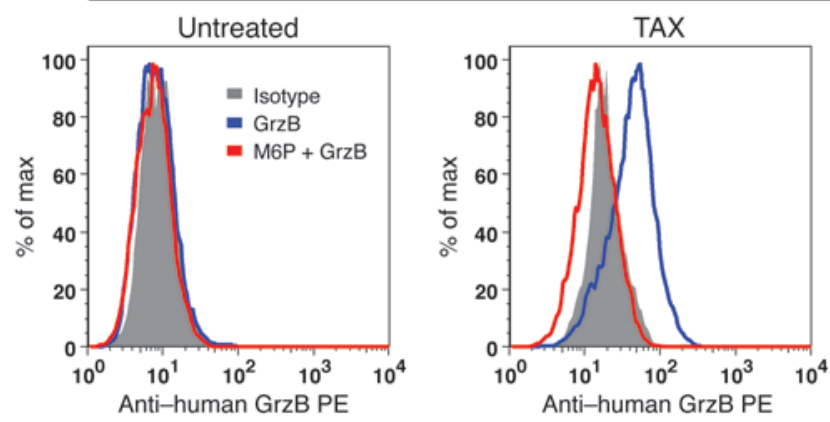

$86 \mathrm{M} 1$ cells
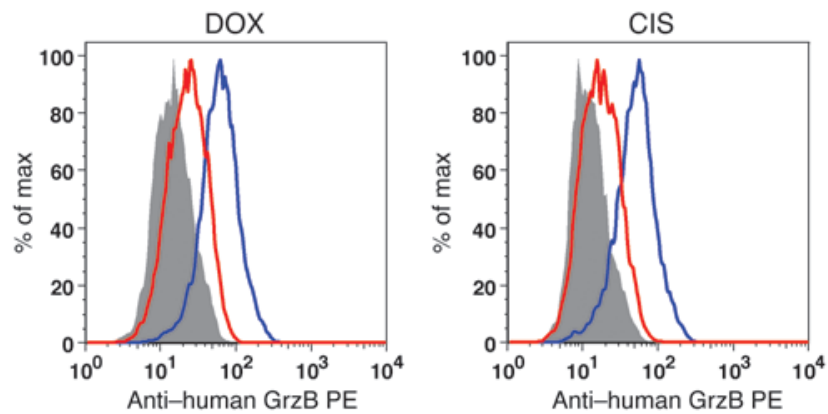

C
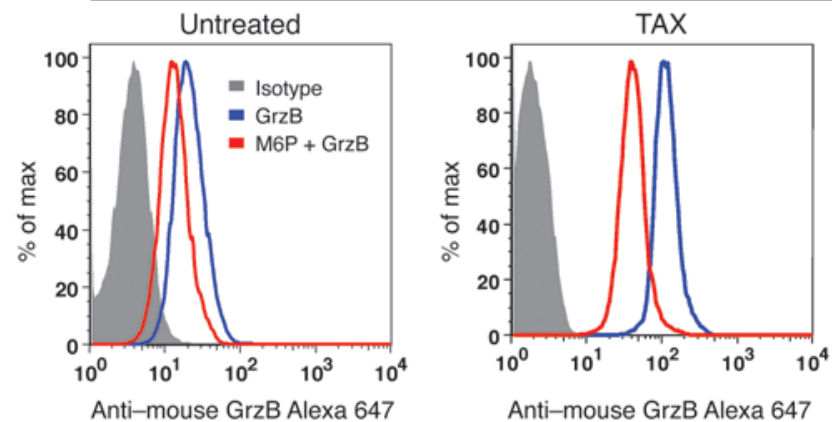

EL-4 cells
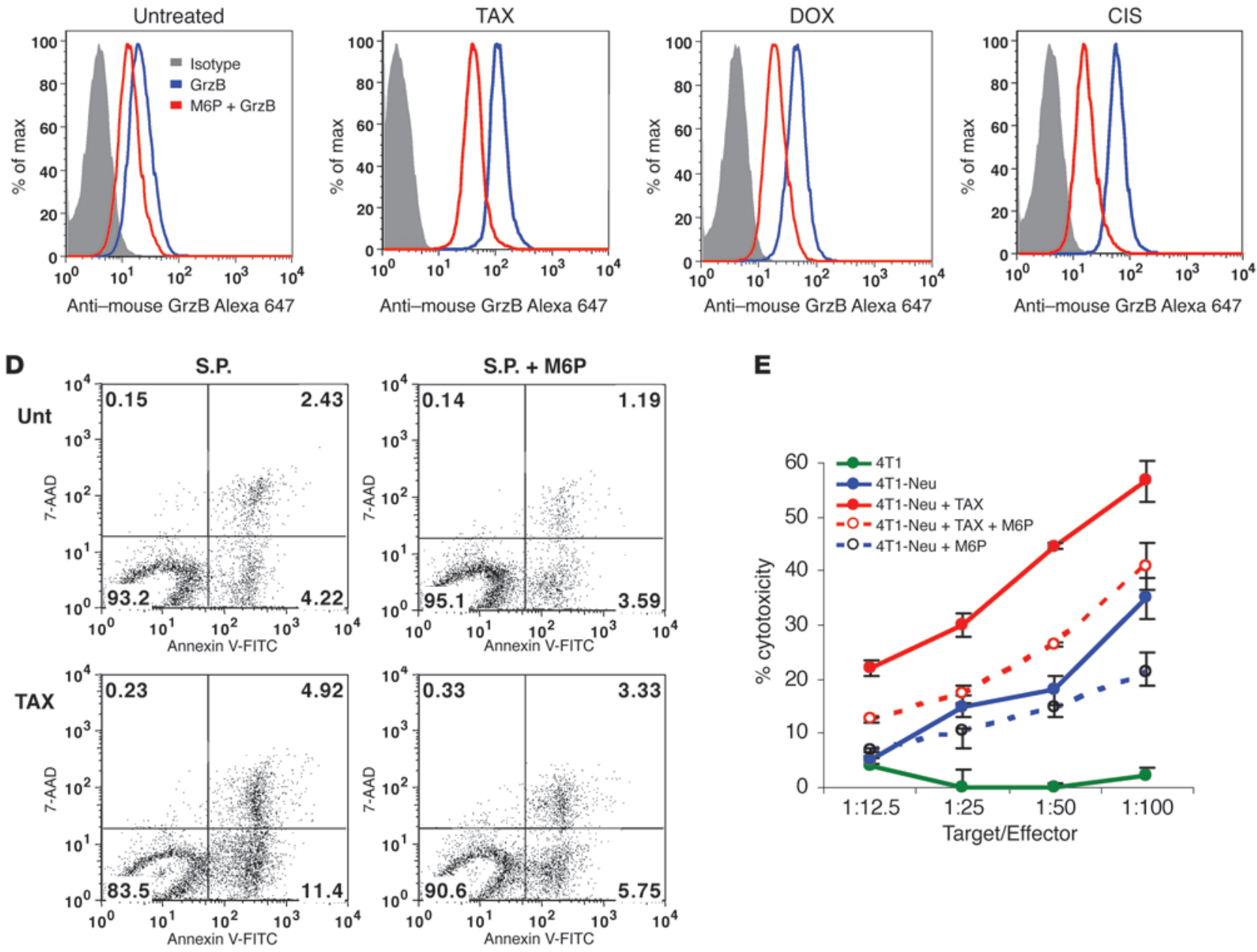

E

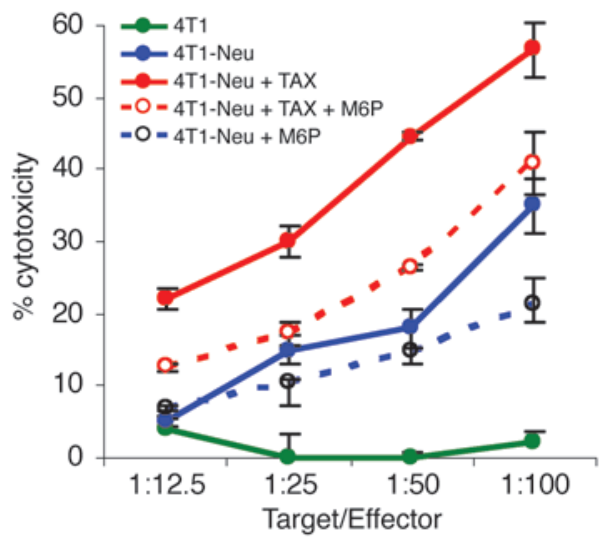




\section{Figure 6}

The role of MPR in the synergistic effect of chemotherapy and CTLs. (A) EL-4, 4T1, or $86 \mathrm{M} 1$ tumor cells were treated with $12.5 \mathrm{nM} \mathrm{TAX}$, $25 \mathrm{ng} / \mathrm{ml} \mathrm{CIS}$, or $25 \mathrm{ng} / \mathrm{ml}$ DOX for 18 hours. Cells were washed, blocked with $10 \%$ mouse or human serum for 20 minutes at $4^{\circ} \mathrm{C}$, and incubated with 1:100 vol/vol cation-independent MPR antibody (Abcam) followed by staining with goat anti-rabbit IgG Alexa Fluor 647. The cells were washed and acquired on a FACSCalibur flow cytometer. The MFI is shown. Data from 1 of 2 experiments with the same results are shown. (B and $\mathbf{C}$ ) To block MPR, M6P (Sigma-Aldrich) was used at a concentration of $20 \mathrm{mM}$. $86 \mathrm{M} 1$ (B) or EL-4 (C) cells were treated with TAX, CIS, or DOX as described above. The cells were incubated with M6P for 15 minutes at room temperature. After 2 washes, the cells were incubated with recombinant human (B) or mouse (C) GrzB for 2 hours. The cells were permeabilized and labeled with anti-mouse GrzB-Alexa 647 or anti-human GrzB-PE. Two experiments with the same results were performed. (D) EL-4 cells were treated with TAX and loaded with control or specific peptides as described in Methods. In the last 15 minutes of peptide loading, one-half of the cells from each treatment group were incubated with $20 \mathrm{mM} \mathrm{M6P}$. The cells were washed and incubated with DDAO-SE-labeled activated OT-1 $T$ cells at a 1:15 ratio. After 1.5 hours, incubated cells were labeled with Annexin V-FITC and 7AAD. DDAO-SE-negative tumor cells were gated and apoptosis measured by flow cytometry. Three experiments with the same results were performed. (E) Neu-specific T cells were generated by immunization of mice as described in Methods and used as effector cells in CTL assay. 4T1 and 4T1-Neu tumor cells were treated with TAX and M6P as described above and used as targets in ${ }^{51} \mathrm{Cr}$ release assay. Experiments were performed in duplicate. Data are presented as mean \pm SEM.

abrogated the effect of TAX on CTL-induced apoptosis of tumor cells (Figure 5D), confirming the critical role of this mechanism in the synergistic effect of chemotherapy and CTLs.

What could be the mechanism by which chemotherapeutic drugs increased GrzB penetration into tumor cells? We performed a kinetic study to determine the earliest time when TAX, CIS, or DOX could induce penetration of recombinant GrzB into tumor cells. Experiments were performed 30 minutes, 3 hours, 6 hours, and 18 hours after start of the treatment. Eighteen hours was the earliest time when all 3 drugs caused increased entrance of GrzB into tumor cells (data not shown). We asked whether chemotherapy made tumor cells porous for other large molecules. To answer this question, we treated EL-4 tumor cells with TAX, DOX, or CIS overnight and then cultured them with OVA-FITC for 30 minutes to 3 hours. Fluorescence intensity was measured in tumor cells. None of the compounds caused an increase in OVA-FITC penetration (data not shown).

Although GrzB entrance into the cytosol of target cells is dependent on perforin, there is evidence supporting the contribution of the cation-independent M6P receptor (MPR) (insulin growth factor receptor II), alone or in a complex with proteoglycan serglycin, in this process $(20,21)$. We evaluated the effect of TAX, DOX, and CIS on the expression of these molecules in mouse EL-4 and $4 \mathrm{~T} 1$ tumor cells as well as in human 86M1 tumor cells. All 3 drugs induced a 2- to 3 -fold increase in the expression of MPR in all cell lines (Figure 6A) but did not affect the expression of serglycin (Supplemental Figure 5).

To test the effect of chemotherapy on normal cells, we treated splenocytes from naive mice and mononuclear cells isolated from healthy volunteers overnight with TAX, CIS, and DOX. No upregulation of MPR or GrzB uptake was detected (Supplemental Figure 6).
To test the possible role of upregulation of MPR in chemotherapy-induced penetration of $\mathrm{GrzB}$, the receptor was blocked with soluble M6P. In both 86M1 (Figure 6B) and EL-4 cells (Figure 6C), blockade of MPR prevented GrzB accumulation in tumor cells caused by all 3 drugs (Figure 6, B and C). To investigate a possible role of MPR in the cytotoxic effect of CTLs, we cultured OT-1 T cells for 1.5 hours with EL-4 tumor cells loaded with specific peptide. As expected, pretreatment of tumor cells with TAX substantially increased tumor cell apoptosis. This effect was almost completely eliminated in the presence of M6P (Figure 6D). To verify this effect in a different experimental system, we used 4T1 mammary carcinoma expressing rat Neu. Neu-specific $\mathrm{T}$ cells were generated in $\mathrm{BALB} / \mathrm{c}$ mice by immunization with Neu-derived peptide. These $\mathrm{T}$ cells recognized 4T1-Neu target cells but not parental 4T1 cells (Figure 6E). Overnight treatment of target cells with TAX significantly $(P<0.05)$ enhanced the cytotoxicity. This effect was abrogated in the presence of M6P (Figure 6E), suggesting that MPR could indeed be responsible for sensitization of tumor cells to CTLs. We also blocked expression of MPR on 4T1 and EL-4 tumor cells using MPR-specific siRNA (Supplemental Figure 7). This resulted in a dramatic reduction in GrzB uptake by these cells caused by overnight incubation with TAX (Supplemental Figure 7).

Chemotherapy allows for bypassing a requirement for antigen recognition by CTLs. Our data demonstrated that chemotherapeutic drugs increased tumor cell permeability to GrzB. GrzB is released by activated CTLs during antigen-specific interaction with tumor cells. During this interaction, entry of GrzB into the cytoplasm of tumor cells depends on the release of perforin by activated CTLs (20). We hypothesized that chemotherapy could cause a "bystander" effect, whereby GrzB released by activated CTLs was able to penetrate tumor cells that did not express specific antigen and thus avoided direct contact with CTLs. To test this hypothesis, we incubated effector OT-1 T cells with 2 target cells mixed at a 1:1 ratio. One was EL-4 cells loaded with specific peptide and left unlabeled; the other was EL-4 cells loaded with control peptide and labeled with ${ }^{51} \mathrm{Cr}$. In this system, chromium release could be detected only from EL-4 cells loaded with control peptide. TAX-treated EL-4 cells loaded with control peptide were not directly recognized by OT-1 CTLs (Figure 3A). No cytotoxicity of chromium-labeled target cells was detected when they were not treated with TAX. However, pretreatment of target cells with TAX resulted in a substantial increase in chromium release (Figure 7A). It was possible that dying peptideloaded tumor cells could release specific peptide that would be then picked up by chromium-labeled tumor cells, and this would make them more sensitive to CTL recognition. To exclude this possibility, we used 2 different models. P66Neu-specific T cells were incubated with chromium-labeled 4T1 tumor cells (that did not express the Neu antigen) and unlabeled 4T1-Neu cells (transfected with Neu). Pretreatment of tumor cells with TAX substantially increased chromium release from antigen-negative target cells (Figure 7B) that otherwise are not recognized by CTLs (Figure 2D). Finally, we used an experimental system with B16F10 target cells deficient for the $\mathrm{H} 2 \mathrm{~K}^{\mathrm{b}}$ molecule and therefore unable to present $\mathrm{H} 2 \mathrm{~K}^{\mathrm{b}}$-restricted peptides. CTLs generated against $\mathrm{H} 2 \mathrm{~K}^{\mathrm{b}}$ epitope derived from tyrosinase-related protein 2 (TRP-2) were used as effector cells. These CTLs recognized wild-type B16 cells but demonstrated very little cytotoxicity against untreated or TAX-pretreated $\mathrm{H} 2 \mathrm{~K}^{\mathrm{b}}$-deficient target cells (Figure 7C). Unlabeled wild-type B16F10 melanoma cells were mixed with chromium-labeled B16F10K ${ }^{\mathrm{b}}$-negative cells 
A

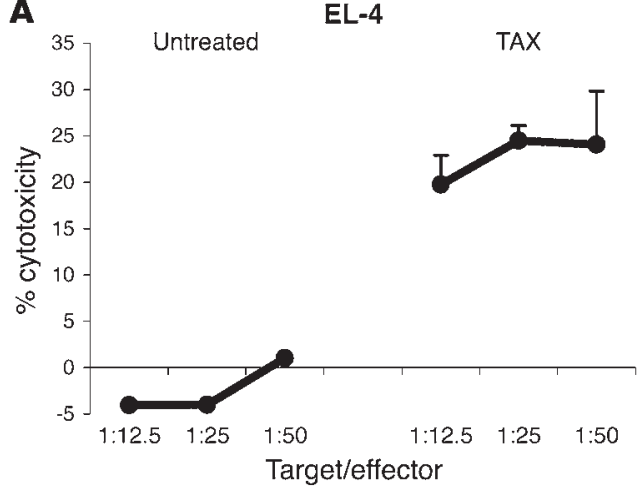

C

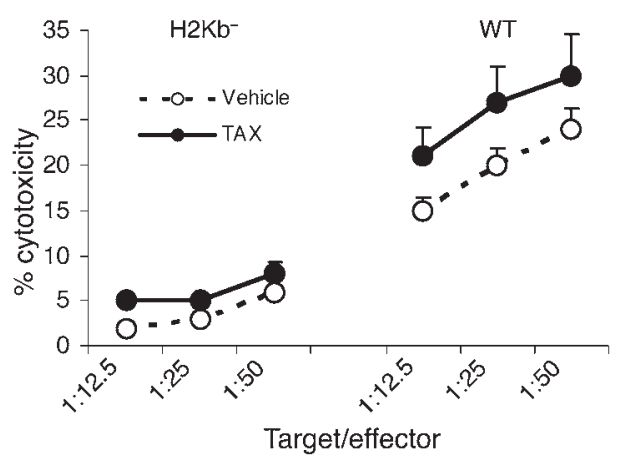

B

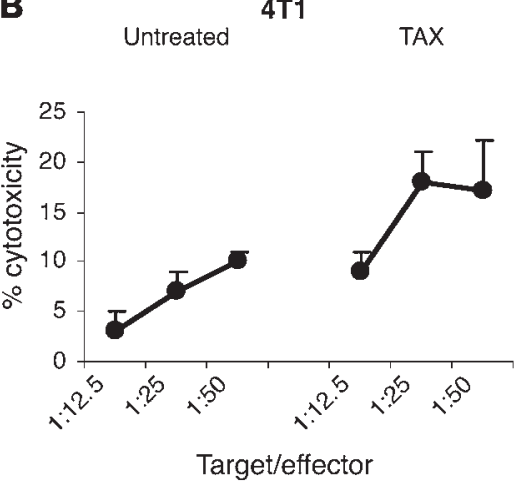

D

Mix of B16F10 WT and H2Kb-

Untreated

TAX

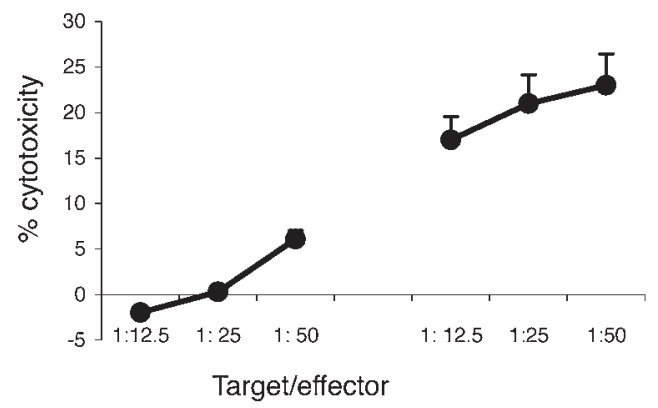

Figure 7

Mechanism of synergistic antitumor activity of CTLs and chemotherapy. (A) EL-4 cells loaded with control peptide and labeled with ${ }^{51} \mathrm{Cr}$ were mixed at a 1:1 ratio with unlabeled EL-4 cells loaded with specific peptide. The mixture of target cells was incubated with OT-1 T cells at the indicated ratios. Pretreatment of target cells with TAX was performed overnight. Standard 4-hour chromium release assay was performed in duplicate. Experiments were repeated 3 times with the same results. Appropriate positive controls were set up with each experiment (data not shown). (B) The experiment was performed essentially as described in Figure 4A. As target cells, chromium-labeled 4T1 cells mixed with unlabeled 4T1-Neu cells were used. Effector T cells were obtained from splenocytes of BALB/c mice immunized with Neu-derived peptide as described in Methods. (C) Wild-type and B16F10 Kb- tumor cells were used as targets in chromium release assay. Labeled target cells treated overnight with TAX were incubated in duplicate with T cells isolated from mice immunized with TRP-2-derived peptide as described in Methods. Two experiments with similar results were performed. (D) Unlabeled wild-type B16F10 tumor cells were mixed at $1: 1$ ratio with ${ }^{51} \mathrm{Cr}$ labeled B16F10 cells with deleted $\mathrm{H}_{2} \mathrm{~K}^{\mathrm{b}}$. These target cells were incubated with T cells from TRP-2-immunized mice, and cytotoxicity was evaluated in standard 4-hour ${ }^{51} \mathrm{Cr}$ release assay. Two experiments with similar results were performed. Data are presented as mean \pm SEM.

and used as targets in the chromium release assay. TRP-2-specific CTLs showed little cytotoxicity against untreated targets; however, pretreatment with TAX dramatically increased the chromium release from $\mathrm{H} 2 \mathrm{~K}^{\mathrm{b}}$-negative cells (Figure 7D).

Thus, pretreatment of tumor cells with TAX allowed CTLs to bypass the requirement for specific recognition to exert their cytotoxic effect by allowing CTL-derived GrzB to penetrate into tumor cells without requirements for antigen-specific cell-cell interaction. If this is indeed the case, then pretreatment of tumor cells with TAX should eliminate the requirement for perforin in CTL-mediated killing. To test this hypothesis, we generated SIINFEKL-specific CTLs by immunization of control and perforin-KO mice with the peptide and then used them as effectors against EL-4 tumor cells loaded with control and specific peptide. As expected, CTLs did not recognize EL-4 cells loaded with control peptide, and in the absence of CTL activation, no cytotoxicity was detected against TAX-treated target cells either (Figure 8A). However, wild-type CTLs recognized and killed EL-4 target cells loaded with specific peptide (Figure 8B). No specific cytotoxicity was detected in perforin-KO CTLs if targets were untreated. However, pretreatment of EL-4 cells with TAX resulted in a substantial level of specific cytotoxicity comparable with the level of wild-type CTLs (Figure 8B). To verify the possible role of this mechanism in vivo, we performed an adoptive transfer experiment, described in Figure 1C, where EG-7 tumor-bearing mice were injected with $\mathrm{T}$ cells from mice immunized with SIINFEKL. Perforin-KO mice developed a high level of antigen-specific $\mathrm{CD}^{+} \mathrm{T}$ cells in response to immunization (Supplemental Figure 8). However, these CTLs failed to provide any antitumor effect after adoptive transfer to an untreated tumor-bearing host. In contrast, the same perforin-KO CTLs showed a substantial antitumor effect when they were transferred into TAX-treated tumor-bearing mice (Figure 8C).

\section{Discussion}

Combination of conventional chemotherapy with cancer vaccines or $\mathrm{T}$ cell adoptive transfer could be an attractive novel therapeutic approach in the treatment of patients with advanced cancer. However, without a clear understanding of how chemotherapy may synergize with immunotherapy, further progress in this field will be limited. Several different mechanisms of such combined effect have been proposed. They may target critical components of immune-suppressive networks such as regulatory T cells (22-24) 
A

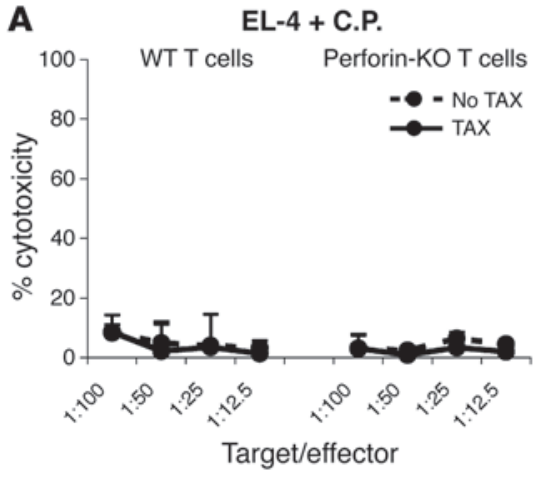

D

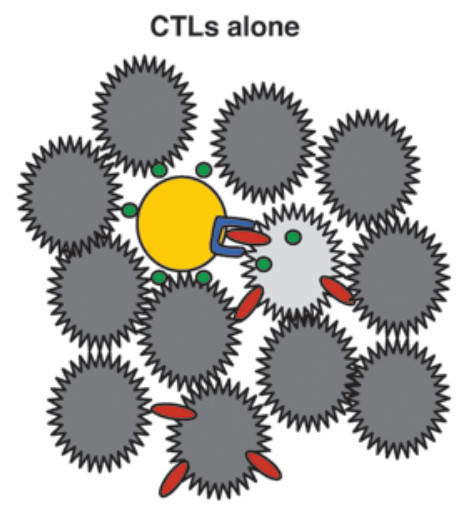

B

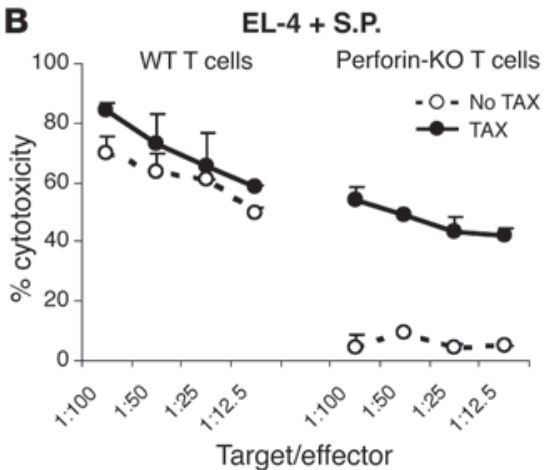

C

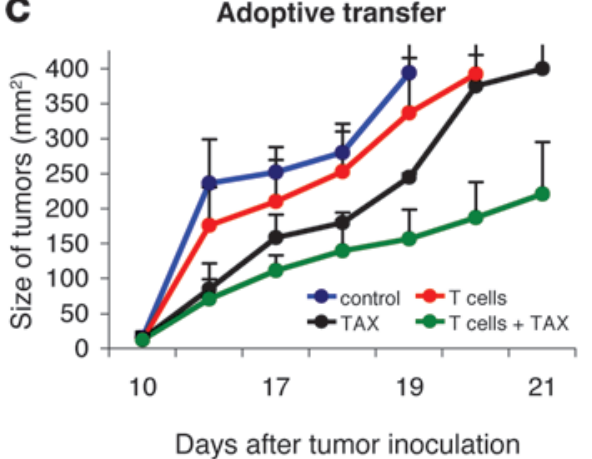

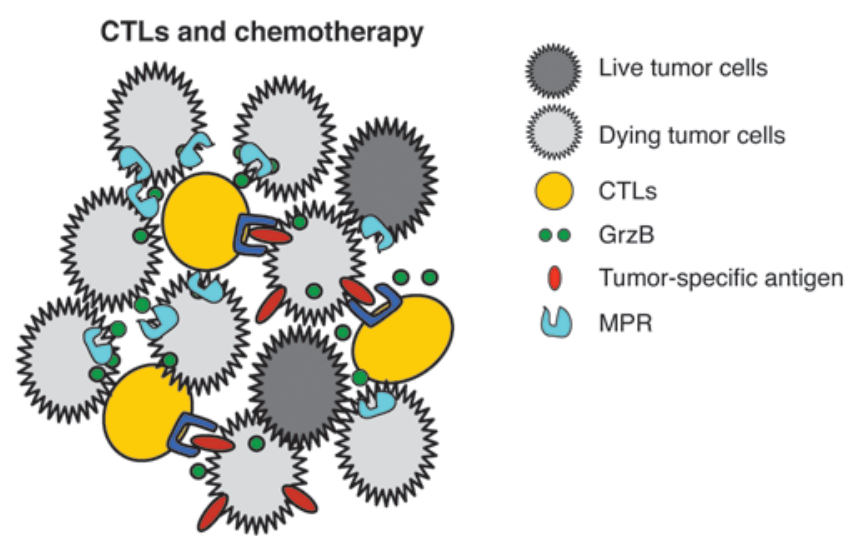

Figure 8

Perforin is not required for CTL activity if targets are treated with chemotherapy. (A and B) Perforin-KO and C57BL/6 mice were immunized with SIINFEKL. Purified T cells from the spleens of these mice were used as effectors in ${ }^{51} \mathrm{Cr}$ release assay. EL-4 cells loaded with control or specific peptide were used as targets. Half of the target cells were pretreated with TAX overnight. Two experiments with the same results were performed. (C) An adoptive transfer experiment using T cells from SIINFEKL-immunized wild-type or perforin-KO mice was performed according to the method described in Figure 1C. The treatment protocol is described in Methods. Tumor size was measured and presented as described in Figure 1C. Data are presented as mean \pm SEM. (D) Schematic representation of the advantage of combination therapy in targeting tumor cells over the use of immunotherapy alone. The figure is a depiction of the possible mechanism of bystander lysis of nonspecific targets within tumors. Red bars indicate the presence of specific antigen on tumor cells.

or myeloid-derived suppressor cells (25) or the expression of inhibitory B7 molecules (26), improved cross-presentation of antigens, etc. $(7,27)$. All these mechanisms could potentially contribute to the effect of combined treatment. They all rely on the persistence, activation, and expansion of antigen-specific T cells after chemotherapy. In several animal models, combination of radiation or chemotherapy with different cancer vaccines indeed resulted in an increased frequency of antigen-specific $T$ cells that was associated with antitumor effects (28-30). However, in mouse experiments, chemotherapy is usually administered only once and does not cause substantial immune suppression. In contrast, conventional chemotherapy in cancer patients is associated with lymphopenia and immune suppression. In mice, repeated injections of TAX also substantially decreased antigen-nonspecific function of $\mathrm{T}$ cells (17). We have observed that even in patients who showed an objective clinical response to chemotherapy administered after vaccination, the antigen-specific $\mathrm{T}$ cell response was abrogated soon after start of the treatment (3). The effect of combined treatment in most of the patients was not long lasting, suggesting that effector cells may not remain functional for a long time. In a different study, the combination of ALVAC-CEA/B7.1 vaccine and systemic chemotherapy did not affect the generation of CEA-specific $T$ cell responses after vaccination (31). In the current study, administration of TAX after adoptive transfer of T cells caused a modest but statistically significant decrease in antigen-specific $T$ cell response. Another known limitation of cancer vaccines is their inability to generate a high frequency of CTLs in cancer patients. This could be due to the fact that most of the epitopes derived from self proteins are weakly immunogeneic, the modes of antigen delivery are not very efficient, and/or the existence of different immune-suppressive mechanisms limits expansion of antigen-reactive clones in tumor-bearing hosts. In the situation where chemotherapy is administered after vaccination, in the absence of continuous vaccination, it is unlikely that this would be sufficient to substantially increase the frequency or affinity of CTLs. All these concerns prompted a search for other potential mechanisms underlying the combined effect of chemotherapy and immunotherapy.

In this study, we used 3 drugs with different mechanism of action. TAX induces apoptosis by affecting microtubules; DOX, by causing DNA damage via intercalation-induced distortion of the double helix and stabilization of the complex formed between DNA and topoisomerase II; and CIS, by crosslinking DNA. We found that all 3 agents sensitized tumor cells to the cytotoxic effect of CTLs. This effect was observed with CTLs spe- 
cific to different antigens. This suggested a common mechanism for this phenomenon. Pretreatment of tumor cells with drugs at doses that did not cause apoptosis was sufficient to cause rapid induction of apoptosis mediated by CTLs. This effect of accelerated killing of tumor cells was antigen specific, since it was not observed when tumor cells were loaded with control antigens and therefore were not recognized by CTLs. Thus, activation of CTLs via antigen-specific interaction with tumor cells was required for the effect of combined treatment. Chemotherapy changed the sensitivity of tumor cells that were not loaded with specific antigens to CTLs. Without chemotherapy, CTLs were not able to recognize and kill target cells that do not express specific antigens. In contrast, after treatment with each of the 3 drugs, these target cells became sensitive to CTLs. Our experiments indicate that this effect was due not to an artifact associated with transfer of antigens from one dying tumor cell to another, but rather to a direct effect of activated CTLs.

How do antigen-specific CTLs kill targets that do not express antigen? Perforin/granzyme and Fas/FasL pathways are 2 major mechanisms by which CTLs destroy target cells. After formation of a synapse between antigen-specific CTLs and targets, CTLs release perforin and serine proteases granzymes. GrzB, a main member of granzyme family, cleaves target cell proteins at specific aspartate residues and triggers caspase activation. It involves primarily proapoptotic "BH3-only" members of the BCL-2 family, such as $\mathrm{BH} 3$-interacting domain death agonist (BID), which results in the leakage of proapoptotic mitochondrial mediators, such as CytC, into the cytosol $(32,33)$.

All tested chemotherapeutic agents did not affect expression of Fas or FasL on tumor cells or splenocytes but caused a dramatic increase in permeability of the cell membrane to GrzB. This suggested that uptake of GrzB may play a major role in sensitization of tumor cells to CTLs. TAX and CTLs utilized different pathways of initial apoptosis activation: CTLs primarily induce release of mitochondrial CytC (which is consistent with the mechanism of GrzB effect), whereas TAX does not cause CytC activation and did not increase CytC activation caused by CTLs. The paradox is that the CytC pathway ultimately targets caspase-3, which is also a target for TAX. Thus, release of CytC alone cannot directly explain why CTLs and TAX have a synergistic effect on induction of apoptosis. However, it is known that several mitochondrial proteins may cause apoptosis in a caspase-independent manner. One of them is EndG $(19,34)$. Our experiments demonstrated that similar to CytC, CTLs caused release and nuclear localization of EndG in tumor cells, whereas TAX did not affect this process. These data suggest a mechanism whereby TAX and CTLs exert a synergistic effect in induction of tumor cell apoptosis.

What could be responsible for increased permeability of tumor cells to GrzB? In both humans and rodents, delivery of granzymes to target cell requires perforin, a pore-forming protein. A direct uptake of granzymes through perforin pores in either the plasma membrane or endosomes is considered to be one of the major mechanisms of granzyme delivery to target cells. More recently, it has been suggested that MPR could be solely responsible for the uptake of GrzB (35). Although this point of view was challenged in subsequent studies $(21,36,37)$, this receptor is considered to be an important factor that together with perforin mediates GrzB entry into the cell. Recently, it has been shown that if cells were incubated with GrzB in the presence of perforin, MPR had minimal impact on GrzB's effect. In contrast, when GrzB was delivered into cells via adenovirus, MPR was directly responsible for GrzB activity (21). Our experiments demonstrated that TAX, DOX, and CIS induced a substantial increase in the expression of MPR on human and mouse tumor cells and that this receptor was primarily responsible for the increased uptake of GrzB by tumor cells treated with chemotherapeutic drugs and for the synergistic effect of CTLs and chemotherapy. These data demonstrate for the first time to our knowledge that chemotherapy may regulate GrzB uptake via upregulation of MPR and thus bypass the requirement for perforin. Indeed, our experiments have shown that perforin-deficient CTLs that were not able to kill nontreated target cells were very effective against tumor cells pretreated with TAX, as well as in adoptive transfer experiments in tumor-bearing mice treated with TAX. This may explain how activated CTLs are able to kill not only target cells that expressed specific antigen but also those that did not.

Our data suggest a mechanism whereby CTLs and chemotherapy exert a combined effect. When cancer vaccines or $\mathrm{T}$ cell transfer protocols are used as a single modality, only limited numbers of $\mathrm{T}$ cells are able to penetrate the tumor parenchyma. CTLs exert their cytotoxic effect only against tumor cells that express specific antigen, since it requires formation of synapse, direct cell-cell contact, and release of perforin and granzymes. Even if GrzB is able to diffuse from the synapse, it does not affect other tumor cells, since it requires perforin for effective penetration into target cells. Thus, the CTL effect is limited by the expression of specific antigen on tumor cells and the presence of different immune-suppressive factors in the tumor microenvironment (38). If chemotherapy is administered immediately after vaccination or $\mathrm{T}$ cell transfer, it causes disruption of tumor stroma that allows for better penetration of antigen-specific T cells. In addition, chemotherapy causes a substantial increase in MPR expression on tumor cells. Small numbers of activated CTLs interacting with tumor cells expressing tumor antigen can release GrzB that can penetrate into neighboring tumor cells without a requirement for cell-cell contact. Therefore, large numbers of tumor cells including those that do not express specific antigen would be susceptible to the effect of CTLs (Figure 8D). This may explain the substantial enhancement of the antitumor effect of combined treatment. This effect is limited to the cells that are sensitive to chemotherapy. Therefore, it should not increase nonspecific toxicity of conventional chemotherapy, as has been demonstrated in all reported clinical trials to date $(2-6,31)$. It is likely that this effect would not be long lasting, since CTL activity will be eliminated by chemotherapy and the immune-suppressive tumor microenvironment. However, it may provide a sufficient window to achieve a significant antitumor effect. Since memory T cells are more resistant to chemotherapy than effector T cells (39), it is possible that subsequent immunization would be able to boost antitumor immunity and thus provide a longer-lasting effect of combined therapy. This provides a rationale for treatment of advanced-stage cancer patients by combining standard-of-care chemotherapy with relatively nontoxic and highly specific immunotherapy.

\section{Methods}

Mice and tumor models. Female C57BL/6J (B6, H-2 $)$, BALB/c (H-2 $\left.{ }^{\mathrm{d}}\right)$, perforin-KO (C57BL/6-Pfp $\left.{ }^{\mathrm{tm} 1 \mathrm{Sd} z}\right)$, and 2C-TCR and OT-1 mice were purchased from The Jackson Laboratory or Charles River Laboratories. Recognized principles of laboratory animal care were followed (Guide for the Care and Use of Laboratory Animals, National Research Council, 1996), and animal 
protocols were approved by the H. Lee Moffitt Cancer Research Institute Animal Care and Use Committee. Murine lymphoblastoma cell line EL-4 and B16-F10 melanoma were purchased from ATCC. B16F10K ${ }^{\mathrm{b}}$-negative cells that do not express the $\mathrm{H} 2 \mathrm{~Kb}$ molecule were isolated as an escape mutant from vaccinated mice. Breast cancer cell lines TUBO, 4T1, and 4T1-Neu (a stably transfected tumor cell line that expresses Neu antigens) were described previously Tumor cell lines were treated with $12.5 \mathrm{nM}$ TAX, $12.5 \mathrm{ng} / \mathrm{ml}$ DOX, and $12.5 \mathrm{ng} / \mathrm{ml}$ CIS for 18 hours prior to use as targets in various assays.

Peptides used in these studies were OVA-derived H2 $\mathrm{K}^{\mathrm{b}}$ - SIINFEKL; 2C-specific H2 $\mathrm{K}^{\mathrm{b}}$-SIYRYYGL; p66, Neu-derived H2D $\mathrm{D}^{\mathrm{d}}$ - TYVPANASL; TRP-2-derived H2K $\mathrm{K}^{\mathrm{b}}$ - SVYDFFVWL.

MC38 tumors were established in C57BL/ 6 mice by subcutaneous injection of $3.5 \times 10^{5}$ tumor cells. On day 3 after tumor inoculation, mice were split into 4 groups. Mice were immunized with $5 \times 10^{5} \mathrm{DCs}$ infected with recombinant adenovirus containing the mouse wild-type p53 gene as described elsewhere (40). Immunizations were repeated twice on days 10 and 17 . Mice were treated with TAX $(12.5 \mathrm{mg} / \mathrm{kg}) 3$ days after the second immunization. For the mammary carcinoma TUBO model, tumors were established in $\mathrm{BALB} / \mathrm{c}$ mice by s.c. injection of $2.5 \times 10^{5}$ cells. Mice were immunized 3 times at 7 -day intervals with DCs loaded with Neuderived 066 peptide.

Immunization of mice. Plasmids encoding chicken OVA and the amino terminus of Neu have been previously described (41). Both constructs were in a pcDNA3 backbone and were confirmed by DNA sequencing. Mice were immunized with a total of $100 \mu \mathrm{g}$ of plasmid DNA injected into 2 sites in the quadriceps femoris muscles. Immunization was followed immediately by electroporation of the injected area $(95 \mathrm{~V}, 4$ pulses of $65 \mathrm{~ms}$ with repoling) using an Electro Square Porator device (BTX, model TX830). For peptide immunization, $100 \mu \mathrm{g}$ of synthetic peptide, TLRL (50 $\mu \mathrm{g}$ of poly-IC), and $50 \mu \mathrm{g}$ of agonistic anti-CD40 (clone FGK45.5) were injected i.v. as a cocktail mixture. Poly-IC (a stabilized formulation containing poly-L-lysine and carboxymethyl cellulose, poly-ICLC, or Hiltonol) was a gift from Andres Salazar (Oncovir Inc., Washington, DC).

Detection of apoptosis, cleaved caspase-3, and CytC. Untreated and TAXtreated tumor cells were labeled with 0.6 $\mu \mathrm{M}$ DDAO-SE (Molecular Probes, Invitrogen) and loaded with $0.1 \mu \mathrm{g} / \mathrm{ml}$ of the control or specific peptide for 1 hour. Anti-cleaved caspase-3, PE-labeled (reactive against both human and mouse forms), was purchased from BD Biosciences. DDAO-SE-labeled target cells were mixed with effector cells at a 1:5 or $1: 10$ ratio in round-bottom tubes (42). The mixtures were incubated at $37^{\circ} \mathrm{C}, 5 \% \mathrm{CO}_{2}$ for 15 minutes or 30 minutes. The cells were washed, fixed, and permeabilized with Fix/Perm solution (BD Biosciences) and then stained for 60 minutes on ice with $15 \mu$ l PE-labeled anti-cleaved caspase-3 monoclonal antibody, followed by flow cytometric analysis. For CytC detection, cells were permeabilized using digitonin buffer for 5 minutes at $4{ }^{\circ} \mathrm{C}$. The cells were then fixed using $4 \%$ paraformaldehyde and blocked with $3 \%$ BSA in PBS containing $0.05 \%$ saponin for 60 minutes. The cells were later labeled with mouse monoclonal anti-CytCFITC antibody (Abcam Inc.) for 60 minutes and cells analyzed by flow cytometry. For detection of apoptosis, the effector cells were labeled with DDAO-SE and incubated with target cells at a 20:1 ratio for various periods of time. Cells then were stained with Annexin V-FITC (BD Biosciences) and the viability dye 7-AAD. The proportion of Annexin $\mathrm{V}$-positive cells was measured within the population of tumor cells by flow cytometry. All cells prepared for flow cytometry were analyzed using FACSCalibur instrumentation (BD).

Staining for EndG. EL-4 cells were treated with TAX for 16 hours and loaded with control or specific peptide. The target cells were incubated for 1 hour with activated Po-Pro-3 iodide-labeled (Invitrogen) OT-1 T cells at a 1:10 ratio. Slides were prepared, and cells were fixed and labeled for EndG as described previously (19). Micrographs were taken with a Leica TCS SP5 AOBS laser scanning confocal microscope through a $63 \times / 1.40$ NA Plan Apochromat oil immersion objective lens (Leica Microsystems). 405 Diode and argon 488 laser lines were applied to excite the samples, and tunable emissions were used to minimize crosstalk between fluorochromes. Image sections at $0.5 \mu \mathrm{m}$ were captured with photomultiplier detectors, and maximum projections were prepared with LAS AF software version 2.1.0 (Leica Microsystems). Volume-rendered images were created using Imaris version 5.0.3 (Bitplane). Intensity analysis was performed using Image Pro Plus version 6.2 (Media Cybernetics Inc.).

Staining of tumor cells for GrzB. EL-4 target cells were treated with $12.5 \mathrm{nM}$ TAX for 16 hours prior to the assay. The cells from both untreated and TAX-treated groups were loaded with either control or specific peptide $(0.1 \mu \mathrm{g} / \mathrm{ml})$ for 30 minutes and then labeled with CellTracker Blue CMAC (7-amino-4-chloromethylcoumarin) (Invitrogen). Target cells were incubated with effector OT-1 T cells at a 1:10 ratio. After 7-60 minutes incubation, cells were fixed on slides with a mixture of methanol and acetone and incubated with mouse anti-GrzB monoclonal antibody at a 1:200 dilution for 1 hour. Secondary antibody (rat anti-mouse FITC-conjugated IgG) was used for labeling the cells. The slides were washed, dried, and mounted with anti-fade mount and observed under a Zeiss fluorescence microscope. The percentage of GrzB-positive target cells was calculated among tumor cells in each group. MPR antibody was purchased from Abcam, and M6P from Sigma-Aldrich.

CTL assay. Spleen cells were cultured with $10 \mu \mathrm{g} / \mathrm{ml}$ of specific peptide (as described in Results) for 72 hours. T cells were purified using T cell enrichment columns (R\&D Systems). For 4-hour ${ }^{51} \mathrm{Cr}$ release assays, tumor cells were incubated with control peptide or specific peptide for 30 minutes, labeled with $100 \mu \mathrm{Ci} \mathrm{Na}{ }^{51} \mathrm{CrO}_{4}$ for 60 minutes, washed, and plated into 96-well round-bottom plates at a cell density of $1 \times 10^{3}$ tumor target cells/well. Target cells were incubated with effector $\mathrm{T}$ cells in duplicate at the indicated $\mathrm{E} / \mathrm{T}$ ratios in $200 \mu \mathrm{l}$ culture medium at $37^{\circ} \mathrm{C}$ in a $\mathrm{CO}_{2}$ incubator. The cells were harvested, and percent ${ }^{51} \mathrm{Cr}$ release was measured using a gamma counter. The percent specific lysis was calculated as follows: $100 \times[($ experimental release - spontaneous release $) /$ (maximum release - spontaneous release)]. EL-4, 4T1/4T1-Neu, and $\mathrm{B} 16 \mathrm{~F} 10 / \mathrm{B} 16 \mathrm{~F} 10 \mathrm{~K}^{\mathrm{b}}$-negative cells were used as target cells in bystander lysis assay. One-half of the EL-4 cells were treated with $12.5 \mathrm{nM}$ TAX for 16 hours. For antigen-specific target cells, EL-4 cells from untreated and TAX-treated groups were preincubated with $0.1 \mu \mathrm{g} / \mathrm{ml}$ specific peptide for 60 minutes. For bystander cells, untreated and TAX-treated cells were labeled with $100 \mu \mathrm{Ci} \mathrm{Na}{ }^{51} \mathrm{CrO}_{4}$ for 30 minutes. After 3 washes, cells from the untreated antigen-bearing target group $\left(5 \times 10^{3}\right)$ and untreated antigen-free bystander $\mathrm{Cr}^{51}$-labeled $\left(5 \times 10^{3}\right)$ groups were combined at a ratio of 1:1. The same ratio was followed for TAX-treated target and bystander cells. The $\mathrm{CD}^{+} \mathrm{T}$ cells were obtained by negative selection of activated OT-1/2C-TCR splenocytes on T cell enrichment columns

Human tumor samples, tumor cell lines, and cytotoxicity assay. Tumor samples were collected from patients with non-SCLC undergoing surgical resection at the H. Lee Moffitt Cancer Center after informed consent was obtained. Tissue collection and animal procedures were approved by the Institutional Review Board and the Animal Care and Use Committee of the University of South Florida. Tumor samples obtained from patients at the time of surgery were cut into 2 - to $3-\mathrm{mm}^{3}$ pieces and implanted subcutaneously in 6-week-old female $n u / n u$ mice. Patients' tumors propagated in mice were excised and used for the ex vivo studies.

SCLC cell lines H332, DMS, and 86M1 were purchased from ATCC. The cells were maintained in RPMI 1640 media containing 10\% FBS. PBMCs were isolated from healthy donors and were cultured with SCLC H332 
cell lysate at a cell equivalent ratio of 20:1 for 5-7 days. PBMCs were then harvested and used as effector cells in cytotoxicity assay. Cytolysis was measured using a standard 6-hour chromium release assay. H332 SCLC target cells or PBMCs were cultured in the presence of $100 \mathrm{nM}$ TAX or left untreated for 18 hours prior to being used as target or effector cells. Target cells were washed, labeled with $100 \mu \mathrm{Ci} \mathrm{Na}{ }^{51} \mathrm{CrO}_{4}$ for 1 hour in PBS, washed, and plated in U-bottom 96-well plates. Different concentrations of the effector cells were added in duplicate to generate $\mathrm{E} / \mathrm{T}$ ratios of 100:1, 50:1, and 25:1.

Statistics. Statistical analysis was performed using 2-tailed Mann-Whitney $U$ or Wilcoxon nonparametric tests, with significance determined at $P<0.05$. Tumor measurements were analyzed using 2 -way ANOVA test with Bonferroni post-hoc test. All statistical analyses were performed using GraphPad Prism 5 (GraphPad Software).

1. Rosenberg SA, Yang JC, Restifo NP. Cancer immunotherapy: moving beyond current vaccines. Nat Med. 2004;10(9):909-915.

2. Gribben JG, et al. Unexpected association between induction of immunity to the universal tumor antigen CYP1B1 and response to next therapy. Clin Cancer Res. 2005;11(12):4430-4436.

3. Antonia SJ, et al. Combination of p53 cancer vaccine with chemotherapy in patients with extensive stage small cell lung cancer. Clin Cancer Res. 2006; 12(3 Pt 1):878-887.

4. Arlen PM, et al. A randomized phase II study of concurrent docetaxel plus vaccine versus vaccine alone in metastatic androgen-independent prostate cancer. Clin Cancer Res. 2006;12(4):1260-1269.

5. Schlom J, Arlen PM, Gulley JL. Cancer vaccines: moving beyond current paradigms. Clin Cancer Res. 2007;13(13):3776-3782.

6. Wheeler CJ, Das A, Liu G, Yu JS, Black KL. Clinical responsiveness of glioblastoma multiforme to chemotherapy after vaccination. Clin Cancer Res. 2004; 10(16):5316-5326.

7. Ramakrishnan R, Antonia S, Gabrilovich DI. Combined modality immunotherapy and chemotherapy: a new perspective. Cancer Immunol Immunother. 2008; 57(10):1523-1529.

8. Apetoh L, et al. Toll-like receptor 4-dependent contribution of the immune system to anticancer chemotherapy and radiotherapy. Nat Med. 2007; 13(9):1050-1059.

9. Zitvogel L, Apetoh L, Ghiringhelli F, Kroemer G Immunological aspects of cancer chemotherapy. Nat Rev Immunol. 2008;8(1):59-73.

10. Disis ML, Bernhard H, Jaffee EM. Use of tumourresponsive T cells as cancer treatment. Lancet. 2009; 373(9664):673-683.

11. Noguchi Y, Chen Y, Old L. A mouse mutant p53 product recognized by CD4+ and CD $8+T$ cells. Proc Natl Acad Sci U S A. 1994;91(8):3171-3175.

12. Gabrilovich DI, Cunningham HT, Carbone DP. IL-12 and mutant $\mathrm{p} 53$ peptide-pulsed dendritic cells for the specific immunotherapy of cancer.J Immunother Emphasis Tumor Immunol. 1996;19(6):414-418.

13. Hilburger Ryan M, Abrams SI. Characterization of CD8+ cytotoxic T lymphocyte/tumor cell interactions reflecting recognition of an endogenously expressed murine wild-type p53 determinant. Cancer Immunol Immunother. 2001;49(11):603-612.

14. Nikitina EY, et al. An effective immunization and cancer treatment with activated dendritic cells transduced with full-length wild-type p53. Gene Ther. 2002;9(5):345-352.

\section{Acknowledgments}

This work was supported by Department of Defense grant BC061707R to D.I. Gabrilovich and by NIH grant R01CA103921 to E. Celis. This work was supported by the microscopy core of H. Lee Moffitt Cancer Center. We thank M. Cotter for help in preparation of the manuscript.

Received for publication August 24, 2009, and accepted in revised form January 13, 2010.

Address correspondence to: Dmitry I. Gabrilovich, H. Lee Moffitt Cancer Center, MRC 2067, 12902 Magnolia Dr., Tampa, FL 33612. Phone: 813.745.6863; Fax: 813.745.1328; E-mail: dmitry. gabrilovich@moffitt.org.

15. Boggio $\mathrm{K}$, et al. Interleukin 12 -mediated prevention of spontaneous mammary adenocarcinomas in two lines of Her-2/neu transgenic mice. J Exp Med. 1998;188(3):589-596.

16. Nava-Parada P, Forni G, Knutson KL, Pease LR, Celis E. Peptide vaccine given with a Toll-like receptor agonist is effective for the treatment and prevention of spontaneous breast tumors. Cancer Res. 2007; 67(3):1326-1334

17. Yu B, et al. Effective combination of chemotherapy and dendritic cell administration for the treatmen of advanced-stage experimental breast cancer. Clin Cancer Res. 2003;9(1):285-294.

18. Pisarev V, et al. Full-length dominant-negative survivin for cancer immunotherapy. Clin Cancer Res. 2003;9(17):6523-6533.

19. van Loo G, et al. Endonuclease G: a mitochondrial protein released in apoptosis and involved in caspase-independent DNA degradation. Cell Death Differ. 2001;8(12):1136-1142.

20. Bolitho P, Voskoboinik I, Trapani JA, Smyth MJ. Apoptosis induced by the lymphocyte effector molecule perforin. Curr Opin Immunol. 2007;19(3):339-347.

21. Veugelers K, Motyka B, Goping IS, Shostak I, Sawchuk T, Bleackley RC. Granule-mediated killing by granzyme B and perforin requires a mannose 6 phosphate receptor and is augmented by cell surface heparan sulfate. Mol Biol Cell. 2006;17(2):623-633.

22. Zou W. Regulatory T cells, tumour immunity and immunotherapy. Nat Rev Immunol. 2006; 6(4):295-307.

23. Vicari AP, et al. Paclitaxel reduces regulatory $\mathrm{T}$ cell numbers and inhibitory function and enhances the anti-tumor effects of the TLR9 agonist PF-3512676 in the mouse. Cancer Immunol Immunother. 2009; 58(4):615-628.

24. Zhang L, et al. Differential impairment of regulatory $T$ cells rather than effector $T$ cells by paclitaxel-based chemotherapy. Clin Immunol. 2008;129(2):219-229.

25. Gabrilovich DI, Nagaraj S. Myeloid-derived suppressor cells as regulators of the immune system. Nat Rev Immunol. 2009;9(3):162-174.

26. Zou W, Chen L. Inhibitory B7-family molecules in the tumour microenvironment. Nat Rev Immunol. 2008; 8(6):467-477.

27. Emens LA, Jaffee EM. Leveraging the activity of tumor vaccines with cytotoxic chemotherapy. Cancer Res. 2005;65(18):8059-8064.

28. Tseng CW, et al. Low-dose radiation enhances therapeutic HPV DNA vaccination in tumor-bearing hosts. Cancer Immunol Immunother. 2009;58(5):737-748.

29. Garnett CT, Schlom J, Hodge JW. Combination of docetaxel and recombinant vaccine enhances T-cell responses and antitumor activity: effects of docetaxel on immune enhancement. Clin Cancer Res. 2008; 14(11):3536-3544.

30. Han HD, et al. A chitosan hydrogel-based cancer drug delivery system exhibits synergistic antitumor effects by combining with a vaccinia viral vaccine. Int J Pharm. 2008;350(1-2):27-34.

31. Kaufman HL, et al. Combination chemotherapy and ALVAC-CEA/B7.1 vaccine in patients with metastatic colorectal cancer. Clin Cancer Res. 2008; 14(15):4843-4849.

32. Sutton VR, et al. Initiation of apoptosis by granzyme B requires direct cleavage of bid, but not direct granzyme B-mediated caspase activation. J Exp Med. 2000;192(10):1403-1414.

33. Heibein JA, et al. Granzyme B-mediated cytochrome $\mathrm{c}$ release is regulated by the Bcl-2 family members bid and Bax. J Exp Med. 2000;192(10):1391-1402.

34. Bajt ML, Cover C, Lemasters JJ, Jaeschke H. Nuclear translocation of endonuclease $\mathrm{G}$ and apoptosisinducing factor during acetaminophen-induced liver cell injury. Toxicol Sci. 2006;94(1):217-225.

35. Motyka B, et al. Mannose 6-phosphate/insulinlike growth factor II receptor is a death receptor for granzyme B during cytotoxic T cell-induced apoptosis. Cell. 2000;103(3):491-500.

36. Trapani JA, et al. A clathrin/dynamin- and mannose-6-phosphate receptor-independent pathway for granzyme B-induced cell death. J Cell Biol. 2003; 160(2):223-233.

37. Dressel R, et al. Granzyme-mediated cytotoxicity does not involve the mannose 6-phosphate receptors on target cells. J Biol Chem. 2004;279(19):20200-20210.

38. Rabinovich GA, Gabrilovich D, Sotomayor EM. Immunosuppressive strategies that are mediated by tumor cells. Annu Rev Immunol. 2007;25:267-296.

39. Nistico P, et al. Chemotherapy enhances vaccine-induced antitumor immunity in melanoma patients. Int J Cancer. 2009;124(1):130-139.

40. Gabrilovich DI, Ishida T, Nadaf S, Ohm J, Carbone DP. Antibodies to vascular endothelial growth factor enhance the efficacy of cancer immunotherapy by improving endogenous dendritic cell function. Clin Cancer Res. 1999;5(10):2963-2970.

41. Cho HI, Niu G, Bradley N, Celis E. Optimized DNA vaccines to specifically induce therapeutic CD8 T cell responses against autochthonous breast tumors. Cancer Immunol Immunother. 2008;57(11):1695-1703.

42. He B, et al. Blockade of Wnt-1 signaling induces apoptosis in human colorectal cancer cells containing downstream mutations. Oncogene. 2005;24(18):3054-3058. 